(2021), 0, 0, pp. 1-37

doi:10.1093//output

\title{
A Phylogenetic Approach to Inferring the Order in Which Mutations Arise during Cancer Progression
}

\author{
YUAN GAO* \\ Division of Biostatistics, The Ohio State University, 1958 Neil Ave, Columbus, OH 43210, US \\ gao.957@osu.edu \\ JEFF GAITHER \\ Institute for Genomic Medicine, Nationwide Children's Hospital, 700 Childrens Dr., Columbus,OH 43205, \\ JULIA CHIFMAN \\ Dept of Mathematics and Statistics, American University, 3501 Nebraska Ave NW, Washington 20016, US \\ LAURA KUBATKO \\ Mathematical Biosciences Institute, The Ohio State University, 1735 Neil Ave, Columbus,OH 43210,US \\ Depts of Statistics and Evolution, Ecology, and Organismal Biology, The Ohio State University, 1958 Neil \\ Ave, Columbus, $O H$ 43210, US
}

2 Although the role of evolutionary processes in cancer progression is widely accepted, increasing attention

3 is being given to evolutionary mechanisms that can lead to differences in clinical outcome. Recent studies

4 suggest that the temporal order in which somatic mutations accumulate during cancer progression is im-

5 portant. Single-cell sequencing provides a unique opportunity to examine the mutation order during cancer

6 progression. However, the errors associated with single-cell sequencing complicate this task. We propose a

7 new method for inferring the order in which somatic mutations arise within a tumor using noisy single-cell

8 sequencing data that incorporates the errors that arise from the data collection process. Using simulation, we

*To whom correspondence should be addressed. 
9 show that our method outperforms existing methods for identifying mutation order in most cases, especially

10 when the number of cells is large. Our method also provides a means to quantify the uncertainty in the

11 inferred mutation order along a fixed phylogeny. We apply our method to empirical data for colorectal and

12 prostate cancer.

Key words: Bayesian inference; Cancer evolution; Error effects quantification; Mutation order; Single-cell sequencing.

15 Cancer progression is a dynamic evolutionary process that occurs among the individual cells within each

16 patient's tumor. Cancer develops from a single cell in normal tissue whose genetic alterations endow a growth

17 advantage over the surrounding cells, allowing that cell to replicate and to expand, resulting in the formation

18 of a clonal population of identical cells. Cells within this clonal population may then undergo their own

19 somatic mutations, followed by replication and formation of subclones. During this complex process, many

20 competitive and genetically diverse subpopulations may be formed, resulting in intratumoral heterogeneity

21 (ITH) depicted in Fig. 1(a) (O'Sullivan and others, 2003; Ishwaran and others, 2009; Jamal-Hanjani and others, 2017; Ascolani and Liò, 2019). Ortmann and others (2015) demonstrate that the type of malignancy and the response to treatment of myeloproliferative neoplasm patients are affected by the order in which somatic mutations arose within the patients' tumors. Though this study is specific to one type of cancer, the timing and organization of somatic mutations are crucial to clinical outcomes for other cancers as well. Determining the temporal order of mutations required for tumor progression is thus critical, especially in the field of targeted therapeutics. However, this information cannot be observed directly, since genomic data is most often collected at one snapshot in time. Consequently, use of computational methods that infer the order of mutations from DNA sequence data is the approach of choice.

Most studies on cancer phylogenetics utilize bulk high-throughput sequencing data, but signals from bulk sequencing only reflect the overall characteristics of a population of sequenced cells, rather than the characteristics of individual cells. Variation in the mutational signatures among different cells in a tumor is thus difficult to evaluate from bulk sequencing data. Single-cell sequencing (SCS) data is promising because 4 it enables sequencing of individual cells, thus providing the highest possible resolution available on the mutational history of cancer. However, the high error probabilities associated with SCS data complicate the 
60 We assume that we are given a phylogenetic tree with branch lengths that displays the evolutionary rela61 tionships among a sample of $J$ cells within a tumor. To infer the locations (branches) on which a set of 62 somatic mutations are acquired in the tree, we need to model the evolutionary process of the somatic mu-

development of methods for inference of the mutational history. The whole-genome amplification (WGA) process used to produce SCS data results in a variety of errors, including allelic dropout (ADO) errors, false positives (FP), non-uniform coverage distribution, and low coverage regions. ADO contributes a considerable number of false negatives (FN) in point mutations (Navin, 2014).

Recently, several studies have proposed various mathematical methods to infer mutation order (Fig. 1(c) - Fig. 1(e)) from data arising from single-cell somatic mutations. Of particular interest are the methods of Jahn and others (2016) and Zafar and others (2017), called SCITE and SiFit, respectively. SiFit uses an MCMC approach as a heuristic to find the maximum likelihood tree from imperfect SCS data. Based on the inferred tumor phylogenetic tree, SiFit estimates the mutation order by estimating the most likely mutation status of the tips and the internal nodes using a dynamic programming algorithm. Although both SCITE and SiFit by default output only the order of the mutations, both can be used to account for uncertainty in the inferred order. For example, because SCITE uses an MCMC algorithm for inference, the posterior probability associated with various mutation orders can be obtained by examining the frequency with which these orders are sampled by the MCMC algorithm. Similarly, the authors of SiFit recently developed a method called SiCloneFit (Zafar and others, 2019) that utilizes MCMC to sample trees, and thus the algorithm from SiFit for inferring mutation order on a fixed tree could be applied to a posterior sample of trees to measure the uncertainty in the mutation order that results from uncertainty in the true tumor phylogeny.

In this paper, we propose a novel method for inferring the order in which mutations arise within an individual tumor given SCS data from the tumor at a single time point. Our approach utilizes models for both the mutational process within the tumor and the errors that arise during SCS data collection in a Bayesian framework, thus allowing us to quantify the uncertainty in the inferred mutation orders along a fixed tumor phylogeny. Our approach thus represents a conceptually distinct and practically important extension of earlier methods.

\section{Methods}


70 Consider somatic mutations of interest at $I$ loci across the genome for a sample of $J$ single cells. The $J$ 71 single cells are sampled from different spatial locations (clones) within the tumor. The mutation data can 72 be either binary or ternary. For binary data, 0 denotes the absence of mutation and 1 means that mutation 73 is present, while for ternary data, 0, 1 and 2 represent the homozygous reference (normal), heterozygous 74 (mutation present) and homozygous non-reference (mutation present) genotypes, respectively.

The $I$ somatic mutations evolve along the tumor evolutionary tree $\mathcal{T}$. Each tip in $\mathcal{T}$ represents one single 76 cell $C_{j}$, where $j=1, \ldots, J$. Let $C=\left\{C_{1}, \ldots, C_{J}\right\}$ be the set of the $J$ single cells under comparison. $\mathcal{T}=(T, \mathbf{t})$ 77 includes two parts: the tree topology $T$ and a vector of branch lengths $\mathbf{t}$. The tree topology $T=(V, E)$ is 78 a connected graph without cycles and is composed of nodes and branches, where $V$ is the set of nodes and $79 E$ is the set of branches. Trees are rooted, and the root $r$ represents the common ancestor (a normal cell 80 without somatic mutations) for all the single cells under comparison. In the context of this paper, all the 81 definitions in the following sections will apply to rooted bifurcating trees. There are $2 J-2$ branches in a

\section{$2.1 \quad$ Notation and terminology} rooted bifurcating tree with $J$ tips, i.e., $E=\left\{e_{1}, e_{2}, \ldots, e_{2 J-2}\right\}$. Let $v$ and $w$ be two nodes in the node set $V$ that are connected by the branch $x$ in the branch set $E$ (i.e., $x=\{v, w\}: v$ is the immediate ancestor node of $w$, and $x$ connects $v$ and $w)$. Then the set $U^{x}(w)$, which includes the node $w$ and all nodes descended from 5 in $\mathcal{T}$, is called the clade induced by $w$. The branch $x$ connects the ancestor node $v$ and the clade induced 6 by $w$, and we define branch $x$ as the ancestor branch of clade $U^{x}(w) . E^{x}(w)$ is a subset of $E$ that includes 7 branches connecting nodes in $U^{x}(w)$, and $C^{x}(w)$ are the tips in $U^{x}(w)$.

Let $G_{i j}$ denote the true genotype for the $i^{t h}$ genomic site of cell $C_{j}$. The $i^{\text {th }}$ genomic site will then have 9 a vector $\mathbf{G}_{i} \in\{0,1\}^{J}$ (for binary data) or $\{0,1,2\}^{J}$ (for ternary data) representing its true genotype for all 
90 the $J$ cells represented by the tips in the tree, where $i=1, \ldots, I$. Let $S_{i j}$ denote the observed data for the

$91 i^{t h}$ genomic site of cell $C_{j}$. Due to the technical errors associated with SCS sequencing, the observed data

$92 S_{i j}$ does not always equal the true genotype $G_{i j}$. For both binary and ternary data, the observed state $S_{i j}$

93 might be flipped with respect to the true mutation $G_{i j}$ due to FP or FN. Missing states ("-") or low-quality

94 states ("?") may be present for some genomic sites, as well. Fig. 2 shows an example of true and observed

95 binary genotype data for the mutations in Fig. 1. In Fig. 2, the observed state is highlighted in red color

96 if it is not consistent with the true genotype. The red numbers are those mutations with flipped observed

97 mutation states relative to the true mutation states. The red dash ("-") indicates a missing value and the red

98 question mark ("?") represents a low-quality value.

99 Mathematically, we represent the observed mutation states of the $J$ single cells at $I$ different genomic

100 sites by an $I \times J$ mutation matrix $\mathbf{S}$ for convenience,

$$
\mathbf{S}=\left(\begin{array}{c}
\mathbf{S}_{1} \\
\mathbf{S}_{2} \\
\vdots \\
\mathbf{S}_{I}
\end{array}\right)=\left(\begin{array}{ccc}
S_{11} & \ldots & S_{1 J} \\
S_{21} & \ldots & S_{2 J} \\
\vdots & \ddots & \vdots \\
S_{I 1} & \ldots & S_{I J}
\end{array}\right)
$$

101 Each entry $(i, j)$ denotes the state observed for mutation $i$ in cell $C_{j}$, so $\mathbf{S}_{i}$ gives the observed data for genomic

102 site $i$ as a vector with $J$ values corresponding to the $J$ single cells. Column $j$ represents the mutations of

103 interest for cell $C_{j}$. In $\mathcal{T}$, let $\mathcal{B}$ be the vector of locations (branches) on which the $I$ mutations occur, i.e.,

$104 \mathcal{B}=\left\{B_{1}, \ldots, B_{I}\right\}$, where $B_{i}$ is the branch on which mutation $i$ is acquired. Note that $B_{i}$ takes values in

$105\left\{e_{1}, e_{2}, \ldots, e_{2 J-2}\right\}$.

\subsection{Somatic mutation process}

107 To model the somatic mutation process, we consider continuous-time Markov processes, which we specify by

108 assigning a rate to each possible transition between states. We consider point mutations. Once a mutation $i$

109 is acquired on a branch $x \in E$, all the branches in the set $E^{x}(w)$ will harbor mutation $i$ but those branches

110 in the set $E \backslash\left(x \cup E^{x}(w)\right)$ will not carry this mutation. Specification of the rates of mutation among states

111 allows for flexibility in the modeling procedure.

112 2.2.1 Binary genotype data For binary genotype data, the mutation process can be modeled by the

$1132 \times 2$ instantaneous rate matrix 


$$
\mathcal{Q}_{\lambda}={ }_{1}^{0}\left(\begin{array}{cc}
0 & 1 \\
-\lambda & \lambda \\
0 & 0
\end{array}\right),
$$

114 where $\lambda$ denotes the instantaneous transition rate per genomic site. The transition probability matrix $P(t)$

115 along a branch of length $t$ is then computed by matrix exponentiation of the product of $\mathcal{Q}_{\lambda}$ and the branch

116 length $t$, which gives

$$
P(t)={ }_{1}^{0}\left(\begin{array}{cc}
P_{00}(t) & P_{01}(t) \\
P_{10}(t) & P_{11}(t)
\end{array}\right)={ }_{1}^{0}\left(\begin{array}{cc}
\exp (-\lambda t) & 1-\exp (-\lambda t) \\
0 & 1
\end{array}\right) .
$$

117 Note that $P_{01}(t)$ is the probability that mutation $i$ is acquired along a branch of length $t$. Under this 118 model and recalling that each mutation evolves independently along different branches in $\mathcal{T}$, the marginal 119 probability that mutation $i$ is acquired on branch $x \in E$, denoted by $P\left(B_{i}=x \mid \mathcal{T}, \mathcal{Q}_{\lambda}\right)$, is thus given by

$$
P\left(B_{i}=x \mid \mathcal{T}, \mathcal{Q}_{\lambda}\right)=\frac{\left[\prod_{B \in\left[E \backslash\left(x \cup E^{x}(w)\right)\right]} P_{00}\left(t_{B}\right)\right] P_{01}\left(t_{x}\right)\left[\prod_{B \in E^{x}(w)} P_{11}\left(t_{B}\right)\right]}{\sum_{z \in E}\left(\left[\prod_{B \in\left[E \backslash\left(z \cup E^{z}(h)\right)\right]} P_{00}\left(t_{B}\right)\right] P_{01}\left(t_{z}\right)\left[\prod_{B \in E^{z}(h)} P_{11}\left(t_{B}\right)\right]\right)},
$$

120 where $t_{B}$ is length of branch $B$. In the numerator, the first term is a product of probabilities over all branches

121 without the mutation, the second term is the probability that the mutation is acquired on branch $x$, and the

122 third term is a product of probabilities over all branches with the mutation, i.e., all branches in $E^{x}(w)$. The

123 denominator is needed to create a valid probability distribution over all possible branches, and is obtained

124 by summing the numerator over all valid branches $z \in E$. The $P\left(B_{i}=x \mid \mathcal{T}, \mathcal{Q}_{\lambda}\right)$ term is normalized by the

125 denominator because we exclude two possible outcomes: a mutation is not acquired on any branch in $\mathcal{T}$, or

126 a mutation is acquired more than once on different branches in $\mathcal{T}$.

As an example, Fig. 3 depicts the observed and true binary genotype for mutation $i=1$ shown in

128 Fig. 2. The set of branches is $E=\left\{e_{1}, \ldots, e_{8}\right\}$ and the corresponding set of branch lengths would be

$129 \mathbf{t}=\left\{t_{1}, \ldots, t_{8}\right\}$. If mutation $i$ is acquired on branch $e_{1}$, the cell descending along branch $e_{8}$ will not carry

130 the mutation, while those descending from the blue branches would carry this mutation. The marginal

131 probability that mutation $i=1$ is acquired on branch $e_{1}$ would be proportional to its numerator, i.e.,

$132 P\left(B_{1}=e_{1} \mid \mathcal{T}, \mathcal{Q}_{\lambda}\right) \propto P_{00}\left(t_{8}\right) P_{01}\left(t_{1}\right)\left[P_{11}\left(t_{2}\right) P_{11}\left(t_{3}\right) P_{11}\left(t_{4}\right) P_{11}\left(t_{5}\right) P_{11}\left(t_{6}\right) P_{11}\left(t_{7}\right)\right]$. 
133 2.2.2 Ternary genotype data The mutation model for ternary data is complex and includes three pos-

134 sible ways that mutation $i$ originates on a branch $x$ in $\mathcal{T}$ :

135

140 We let $B_{i}$ be the location at which mutation $i$ originates, $B_{i}^{0 \rightarrow 1}$ would be the branch on which mutation 141 status transitions from 0 to $1, B_{i}^{0 \rightarrow 2}$ is the branch on which mutation status transitions from 0 to 2 , and 142 143 all cells belonging to $C^{x}(w)$ will carry 1 or 2 mutations. In other words, $G_{i j}=1$ or 2 for all $C_{j} \in C^{x}(w)$ and $144 G_{i j}=0$ for all $C_{j} \in C \backslash C^{x}(w)$. We define the instantaneous rate matrix $\mathcal{Q}_{\lambda}$ as

$$
\mathcal{Q}_{\lambda}={ }_{1}\left(\begin{array}{ccc}
0 & 1 & 2 \\
-\left(\lambda_{1}+\lambda_{1} \lambda_{2}\right) & \lambda_{1} & \lambda_{1} \lambda_{2} \\
0 & -\lambda_{2} & \lambda_{2} \\
0 & 0 & 0
\end{array}\right) \text {, }
$$

145 where $\lambda_{1}$ and $\lambda_{2}$ denote the instantaneous transition rate per genomic site of the transitions $0 \rightarrow 1$ and

$1461 \rightarrow 2$, respectively. Studies have provided evidence that direct mutation of $0 \rightarrow 2$ at rate $\lambda_{1} \lambda_{2}$ is possible 147 in principle, although it is extremely rare (Iwasa and others, 2004). If $\lambda_{2}$ is 0 in Expression (2.5), the model 148 will be reduced to the infinite sites diploid model. The transition probability matrix $P(t)=\exp \left(\mathcal{Q}_{\lambda} t\right)$ is then 149 given by

0 $P(t)=1\left(\begin{array}{cc}\exp \left(-\left(\lambda_{1}+\lambda_{1} \lambda_{2}\right) t\right) & \frac{\lambda_{1}\left(\exp \left(-\left(\lambda_{1}+\lambda_{1} \lambda_{2}\right) t\right)-\exp \left(-\lambda_{2} t\right)\right)}{\lambda_{2}-\left(\lambda_{1}+\lambda_{1} \lambda_{2}\right)} \\ 0 & \exp \left(-\lambda_{2} t\right) \\ 0 & 0\end{array}\right.$
2

$$
\left.\begin{array}{c}
\frac{\left(\lambda_{1} \lambda_{2}-\lambda_{2}\right) \exp \left(-\left(\lambda_{1}+\lambda_{1} \lambda_{2}\right) t\right)+\lambda_{1} \exp \left(-\lambda_{2} t\right)}{\lambda_{2}-\left(\lambda_{1}+\lambda_{1} \lambda_{2}\right)}+1 \\
1-\exp \left(-\lambda_{2} t\right) \\
1
\end{array}\right) .
$$

150 The marginal probability that mutation $i$ originates on branch $x \in E$ for these three possible conditions is

151 thus given by 


$$
\begin{gathered}
P\left(B_{i}^{0 \rightarrow 1}=x \mid \mathcal{T}, \mathcal{Q}_{\lambda}\right)=\frac{Q\left(B_{i}^{0 \rightarrow 1}=x\right)}{\sum_{z_{1} \in E}\left[Q\left(B_{i}^{0 \rightarrow 1}=z_{1}\right)+Q\left(B_{i}^{0 \rightarrow 2}=z_{1}\right)+\sum_{z_{2}} Q\left(B_{i}^{0 \rightarrow 1}=z_{1}, B_{i}^{1 \rightarrow 2}=z_{2}\right)\right]}, \\
P\left(B_{i}^{0 \rightarrow 2}=x \mid \mathcal{T}, \mathcal{Q}_{\lambda}\right)=\frac{Q\left(B_{i}^{0 \rightarrow 2}=x\right)}{\sum_{z_{1} \in E}\left[Q\left(B_{i}^{0 \rightarrow 1}=z_{1}\right)+Q\left(B_{i}^{0 \rightarrow 2}=z_{1}\right)+\sum_{z_{2}} Q\left(B_{i}^{0 \rightarrow 1}=z_{1}, B_{i}^{1 \rightarrow 2}=z_{2}\right)\right]}, \\
P\left(B_{i}^{0 \rightarrow 1}=x, B_{i}^{1 \rightarrow 2}=y \mid \mathcal{T}, \mathcal{Q}_{\lambda}\right)=\quad \\
\frac{Q\left(B_{i}^{0 \rightarrow 1}=x, B_{i}^{1 \rightarrow 2}=y\right)}{\sum_{z_{1} \in E}\left[Q\left(B_{i}^{0 \rightarrow 1}=z_{1}\right)+Q\left(B_{i}^{0 \rightarrow 2}=z_{2}\right)+\sum_{z_{2}} Q\left(B_{i}^{0 \rightarrow 1}=z_{1}, B_{i}^{1 \rightarrow 2}=z_{2}\right)\right]}
\end{gathered}
$$

152 where

$$
Q\left(B_{i}^{0 \rightarrow 1}=x\right)=\left[\prod_{B \in\left[E \backslash\left(x \cup E^{x}(w)\right)\right]} P_{00}\left(t_{B}\right)\right] P_{01}\left(t_{x}\right)\left[\prod_{B \in E^{x}(w)} P_{11}\left(t_{B}\right)\right],
$$

153

$$
Q\left(B_{i}^{0 \rightarrow 2}=x\right)=\left[\prod_{B \in\left[E \backslash\left(x \cup E^{x}(w)\right)\right]} P_{00}\left(t_{B}\right)\right] P_{02}\left(t_{x}\right)\left[\prod_{B \in E^{x}(w)} P_{22}\left(t_{B}\right)\right],
$$

154

$$
\begin{aligned}
Q\left(B_{i}^{0 \rightarrow 1}=x, B_{i}^{1 \rightarrow 2}=y\right)= & {\left[\prod_{B \in\left[E \backslash\left(x \cup E^{x}(w)\right)\right]} P_{00}\left(t_{B}\right)\right] P_{01}\left(t_{x}\right) } \\
& {\left[\prod_{B \in\left[E^{x}(w) \backslash\left(y \cup E^{y}(b)\right)\right]} P_{11}\left(t_{B}\right)\right] } \\
& P_{12}\left(t_{y}\right)\left[\prod_{B \in E^{y}(b)} P_{22}\left(t_{B}\right)\right]
\end{aligned}
$$

155 As for binary data, we normalize the marginal probabilities to exclude scenarios in which mutations are

156 acquired more than once or in which mutations are not acquired in $\mathcal{T}$. As an example, Fig. S1 in the

157 Supplementary Material depicts the same mutation as in Fig. 3, but considers ternary data, leading to the 158 following:

159

160

161

162

163

164
1. The marginal probability that mutation $i$ transitions from $0 \rightarrow 1$ on branch $e_{1}$ is $P\left(B_{i}^{0 \rightarrow 1}=e_{1} \mid \mathcal{T}, \mathcal{Q}_{\lambda}\right) \propto$ $P_{00}\left(t_{8}\right) P_{01}\left(t_{1}\right)\left[P_{11}\left(t_{2}\right) P_{11}\left(t_{3}\right) P_{11}\left(t_{4}\right) P_{11}\left(t_{5}\right) P_{11}\left(t_{6}\right) P_{11}\left(t_{7}\right)\right]$.

2. The marginal probability that mutation $i$ transitions from $0 \rightarrow 2$ on branch $e_{1}$ is $P\left(B_{i}^{0 \rightarrow 2}=e_{1} \mid \mathcal{T}, \mathcal{Q}_{\lambda}\right) \propto$ $P_{00}\left(t_{8}\right) P_{02}\left(t_{1}\right)\left[P_{22}\left(t_{2}\right) P_{22}\left(t_{3}\right) P_{22}\left(t_{4}\right) P_{22}\left(t_{5}\right) P_{22}\left(t_{6}\right) P_{22}\left(t_{7}\right)\right]$

3. The marginal probability that mutation $i$ transitions from $0 \rightarrow 1$ on $e_{1}$, and from $1 \rightarrow 2$ on $e_{3}$ is $P\left(B_{i}^{0 \rightarrow 1}=e_{1}, B_{i}^{1 \rightarrow 2}=e_{3} \mid \mathcal{T}, \mathcal{Q}_{\lambda}\right) \propto P_{00}\left(t_{8}\right) P_{01}\left(t_{1}\right) P_{11}\left(t_{2}\right) P_{12}\left(t_{3}\right) P_{22}\left(t_{4}\right) P_{22}\left(t_{5}\right) P_{22}\left(t_{6}\right) P_{22}\left(t_{7}\right)$. 
165 The probability $P\left(B_{i}^{0 \rightarrow 1}=e_{1}, B_{i}^{1 \rightarrow 2}=e_{3} \mid \mathcal{T}, \mathcal{Q}_{\lambda}\right)$ is the marginal probability that two mutations at the same

166 locus along the genome mutate on two branches $e_{1}$ and $e_{3}$, respectively. After the first mutation occurs on

167 branch $e_{1}$, the second mutation can occur on any branch except $e_{1}$ and $e_{8}$.

169 To account for FPs and FNs in the observed SCS data, our method applies the error model for binary and 170 ternary data from Kim and Simon (2014), Jahn and others (2016), and Zafar and others (2017). Let $\alpha_{i j}$ be

171 the probability of a false positive error and $\beta_{i j}$ be the probability of a false negative error for genomic site $i$ 172 of cell $C_{j}$

173 For binary data, if the true genotype is 0 , we may observe a 1 , which is a false positive error. If the

174 true genotype is 1 , we may observe a 0 , which is a false negative error. The conditional probabilities of the 175 observed data given the true genotype at genomic site $i$ of cell $C_{j}$ are

$$
\begin{aligned}
& S_{i j}=0 \quad S_{i j}=1 \\
& \mathbf{N}^{i j}=G_{i j}=0\left(\begin{array}{cc}
1-\alpha_{i j} & \alpha_{i j} \\
\beta_{i j} & 1-\beta i j
\end{array}\right),
\end{aligned}
$$

176 where $\mathbf{N}_{01}^{i j}=P\left(S_{i j}=1 \mid G_{i j}=0\right)=\alpha_{i j}$, and other entries are defined similarly. Under the assumption that 177 sequencing errors are independent, if mutation $i$ is acquired on branch $x$, we can precisely quantify the effect 178 of SCS technical errors for mutation $i$ as

$$
P\left(\mathbf{S}_{i} \mid B_{i}=x, \mathcal{T}, \mathbf{N}^{i}\right)=\prod_{j=1}^{J} P\left(S_{i j} \mid G_{i j}\right),
$$

179 where $\mathbf{N}^{i}=\left\{\mathbf{N}^{i 1}, \ldots, \mathbf{N}^{i J}\right\}$. Using the example in Fig. 3, the error probability of the observed genotype condi-

180 tioning on the mutation $i=1$ occurring on branch $e_{1}$ would be $P\left(\mathbf{S}_{1} \mid B_{1}=e_{1}, \mathcal{T}, \mathbf{N}^{1}\right)=\mathbf{N}_{11}^{11} \mathbf{N}_{10}^{12} \mathbf{N}_{11}^{13} \mathbf{N}_{10}^{14} \mathbf{N}_{00}^{15}$,

181 where $\mathbf{N}^{1}=\left\{\mathbf{N}^{11}, \ldots, \mathbf{N}^{15}\right\}$ for this binary data example.

182 For ternary data, the conditional probabilities of the observed data given the true genotype are given by

$$
\begin{aligned}
& S_{i j}=0 \quad S_{i j}=1 \quad S_{i j}=2 \\
& \mathbf{N}^{i j}=G_{i j}=0\left(\begin{array}{ccc}
1-\alpha_{i j}-\alpha_{i j} \beta_{i j} / 2 & \alpha_{i j} & \alpha_{i j} \beta_{i j} / 2 \\
\beta_{i j} / 2 & 1-\beta_{i j} & \beta_{i j} / 2 \\
0 & 0 & 1
\end{array}\right) \text {, }
\end{aligned}
$$

183 where $\mathbf{N}_{01}^{i j}=P\left(S_{i j}=1 \mid G_{i j}=0\right)=\alpha_{i j}$, and the other entries are defined similarly. Under the same assump- 
184

185

186

187

188

189

190

191

192

193

194

195

197

198

200

201 202

\section{3}

204

205

206

207

208 Once the observed status matrix $\mathbf{S}=\left[\mathbf{S}_{1} \ldots \mathbf{S}_{I}\right]^{T}$ of the $I$ mutations has been collected, the next step is to

209 infer the branch on which mutation $i$ takes place, conditioning on $\mathbf{S}$. Given the observed data matrix $\mathbf{S}$, the

\subsection{Missing and low-quality data}

In real data, missing and low-quality states are observed and must be taken into account. For each mutation $i$, we exclude cells with missing states, and a subtree $\mathcal{T}_{i}$ from $\mathcal{T}$ is extracted. The number of tips $J_{i}$ in subtree $\mathcal{T}_{i}$ is less than or equal to $J$. Let $E_{i}$ be the set of branches in subtree $\mathcal{T}_{i}$. The probability that mutation $i$ occurs on branch $x$ is then given by $P\left(B_{i}=x \mid \mathcal{T}, \mathcal{Q}_{\lambda}\right)=P\left(B_{i}=x \mid \mathcal{T}_{i}, \mathcal{Q}_{\lambda}\right)$, where $P\left(B_{i}=x \mid \mathcal{T}_{i}, \mathcal{Q}_{\lambda}\right)$ is computed based on branches in the subtree $\mathcal{T}_{i}$, and $P\left(B_{i}=x \mid \mathcal{T}_{i}, \mathcal{Q}_{\lambda}\right)$ is 0 for those branches $x \in E \backslash E_{i}$. We quantify the effect of the SCS technical errors as

$$
P\left(\mathbf{S}_{i} \mid B_{i}=x, \mathcal{T}, \mathbf{N}^{i}\right)=\prod_{j=1}^{J_{i}}\left(\sum_{S_{i j k}} w_{i j k} P\left(S_{i j k} \mid G_{i j k}\right)\right),
$$

where $w_{i j k}$ is the weight for each possible genotype state at a mutation site. For a site with an observed state that is not missing or ambiguous, $w_{i j k}$ is 1 for the observed state and 0 for all other states. For an ambiguous site, we can assign equal weight for each possible state, or we can assign weight based on sequencing information or other biological characteristics.

\subsection{Inferring the location of a mutation in $\mathcal{T}$}


210 tumor phylogenetic tree $\mathcal{T}$, the error probability matrix $\mathbf{N}=\left\{\mathbf{N}^{i j} \mid 1 \leqslant i \leqslant I, 1 \leqslant j \leqslant J\right\}$, and the mutation

211 process $\mathcal{Q}_{\lambda}$, we can assign a posterior probability distribution $P\left(B_{i} \mid \mathbf{S}, \mathcal{T}, \mathbf{N}, \mathcal{Q}_{\lambda}\right)$ to the location of mutation

$212 i$ using Bayes' Theorem,

$$
P\left(B_{i}=x \mid \mathbf{S}, \mathcal{T}, \mathbf{N}, \mathcal{Q}_{\lambda}\right)=\frac{P\left(\mathbf{S}_{i} \mid B_{i}=x, \mathcal{T}_{i}, \mathbf{N}^{i}\right) P\left(B_{i}=x \mid \mathcal{T}_{i}, \mathcal{Q}_{\lambda}\right)}{P\left(\mathbf{S}_{i} \mid \mathcal{T}_{i}, \mathbf{N}^{i}, \mathcal{Q}_{\lambda}\right)}
$$

213 For mutation $i, P\left(B_{i}=x \mid \mathbf{S}_{i}, \mathcal{T}_{i}, \mathbf{N}^{i}, \mathcal{Q}_{\lambda}\right)$ is computed for all $x$ in set $E_{i}$. For example, there are 8 branches in

214 the tree in Fig. 3, so the branch on which mutation $i=1$ occurs, $B_{1}$, can be any of the 8 branches. For the

215 binary example, the posterior probability that mutation $i=1$ occurs on $e_{1}$ is $P\left(B_{1}=e_{1} \mid \mathbf{S}_{1}, \mathcal{T}_{1}, \mathbf{N}^{1}, \mathcal{Q}_{\lambda}\right) \propto$

$216 P_{00}\left(t_{8}\right) P_{01}\left(t_{1}\right)\left[P_{11}\left(t_{2}\right) P_{11}\left(t_{3}\right) P_{11}\left(t_{4}\right) P_{11}\left(t_{5}\right) P_{11}\left(t_{6}\right) P_{11}\left(t_{7}\right)\right] \cdot \mathbf{N}_{11}^{11} \mathbf{N}_{10}^{12} \mathbf{N}_{11}^{13} \mathbf{N}_{10}^{14} \mathbf{N}_{00}^{15}$. In this way, the posterior

$\mathbf{2 1 7}$ probability that the mutation occurs on each of the 8 branches can be computed, giving the probability

218 distribution for the location of mutation $i=1$, i.e. $P\left(B_{1}=x \mid \mathbf{S}_{1}, \mathcal{T}_{1}, \mathbf{N}^{1}, \mathcal{Q}_{\lambda}\right)$ for $x \in\left\{e_{1}, \ldots, e_{8}\right\}$.

219 To summarize this probability distribution, we construct a $(1-\theta) \times 100 \%$ credible set for the location of

220 mutation $i$ as follows. First, the branches are ranked by their posterior probabilities, and then branches are

$\mathbf{2 2 1}$ added to the credible set in the order of decreasing posterior probability until the sum of their probabilities

222

223

224

225

228 We now consider the joint posterior probability distribution of the locations for the $I$ mutations in the 229 sample of $J$ single cells, which is a distribution on a set of cardinality $(2 J-2)^{I}$. Based on the assumption $\mathbf{2 3 0}$ of independence among the $I$ mutations being considered, the posterior distribution for $\mathcal{B}$ is given by

$$
P\left(\mathcal{B} \mid \mathbf{S}, \mathcal{T}, \mathbf{N}, \mathcal{Q}_{\lambda}\right)=\prod_{i=1}^{I} P\left(B_{i} \mid \mathbf{S}_{i}, \mathcal{T}, \mathbf{N}^{i}, \mathcal{Q}_{\lambda}\right)
$$

231 where $\mathbf{N}^{i}=\left\{\mathbf{N}^{i 1}, \ldots, \mathbf{N}^{i J}\right\}$. From this distribution, we can extract information on the ordering of mutations 232 of interest. For example, if we are interested in the order of mutation $i=1$ and mutation $i=2$ in Fig. 2 , 
233 the joint posterior probability distribution that mutation $i=1$ occurs on branch $x \in E$ and mutation $i=2$

234 occurs on branch $y \in E$ can be used to find the probability that mutation $i=1$ occurs earlier in the tree than

235 mutation $y$. Note that $P^{B_{1}=x, B_{2}=y}=P\left(B_{1}=x, B_{2}=y \mid \mathbf{S}, \mathcal{T}, \mathbf{N}, \mathcal{Q}_{\lambda}\right)=P\left(B_{1}=x \mid \mathbf{S}, \mathcal{T}, \mathbf{N}, \mathcal{Q}_{\lambda}\right) \cdot P\left(B_{2}=\right.$

$\left.236 y \mid \mathbf{S}, \mathcal{T}, \mathbf{N}, \mathcal{Q}_{\lambda}\right)$. This joint distribution can be represented in a matrix given by

$$
\begin{aligned}
& B_{1}=e_{1} \quad B_{1}=e_{2} \quad \ldots \quad B_{1}=e_{8} \\
& B_{2}=e_{1}\left(\begin{array}{cccc}
P^{B_{1}=e_{1}, B_{2}=e_{1}} & P^{B_{1}=e_{2}, B_{2}=e_{1}} & \ldots & P^{B_{1}=e_{8}, B_{2}=e_{1}} \\
P_{2}=e_{8} B_{1}=e_{1}, B_{2}=e_{2} & P^{B_{1}=e_{2}, B_{2}=e_{2}} & \ldots & P^{B_{1}=e_{8}, B_{2}=e_{2}} \\
\vdots & \vdots & \ddots & \vdots \\
P^{B_{1}=e_{1}, B_{2}=e_{8}} & P^{B_{1}=e_{2}, B_{2}=e_{8}} & \ldots & P^{B_{1}=e_{8}, B_{2}=e_{8}}
\end{array}\right) .
\end{aligned}
$$

237 Adding entries of the matrix for which branch $e_{1}$ is earlier in the tree than branch $e_{2}$ thus gives the probability

238

239

240

241

243 To evaluate the ability of our method, which we call MO (Mutation Order), to correctly identify the locations

244 and the order of a set of mutations under different conditions, we conduct a series of simulation studies with

245 data simulated under different assumptions. The goal is to assess the effect of data quality (complete or

246 incomplete, high or low error probabilities), number of cells, branch lengths, number of mutations and type

$\mathbf{2 4 7}$ of genotype data on the performance of our method. We consider a total of 12 scenarios, with 100 replicates

248 for each setting within each scenario. Scenarios 1 - 4 involve data generated under our model for either 10

249 cells (scenarios 1 and 2) or 50 cells (scenarios 3 and 4) for either long branch lengths (scenarios 1 and 3 ) or

250 short branch lengths (scenarios 2 and 4) data. Scenarios 5 - 8 consider data simulated under various models

251 implemented in the CellCoal software (Posada, 2020). Scenarios 9 and 10 involve data generated under

252 our model, but with mutations placed on branches with varying (rather than equal) probabilities. Finally,

253 scenarios 11 and 12 consider data simulated under the finite sites assumption (all other simulation settings 
254 used the infinite sites assumption). The methods used to simulate data under these different scenarios are

255

256

\section{$3.1 \quad$ Accuracy of MAP estimates}

258 We assess the accuracy of the MAP estimates in MO across the 100 trees within each simulation setting in

259 several ways, including whether the mutation is inferred to occur on the correct branch ("location accuracy"),

260 whether any pair of mutations are inferred to occur in the correct order ("order accuracy"), and whether a

261 pair of mutations that occur on adjacent branches are inferred to occur in the correct order ("adjacent order

262 accuracy"). In evaluating both the order accuracy and adjacent order accuracy, if two sequential mutations

263 are inferred to occur on the same branch, then it is counted as ordering the mutations incorrectly. In addition,

264 pairs of mutations that occur on the same branch are also included in the computation of order accuracy

265 and adjacent order accuracy. The details of how the MAP estimates are assessed are given in Section B of

266 the Supplementary Material. Tables 1 to 4 in the Supplementary Material show the location accuracy for

267 scenarios 1 to 4 with each cell entry corresponding to a unique setting of $\alpha, \beta$, type of genotype and missing

268 data percentage. In most cases, the location accuracy of MO is high except when the error probabilities are

269 high. Comparing the effect of the size of the tree (i.e., the number of cells), the accuracy of settings with

27050 cells (Tables 3 and 4) is as good as for settings with 10 cells (Tables 1 and 2) in most cases. When error

271 probabilities are large, the accuracy of settings with 50 cells in Tables 3 and 4 are slightly lower than those

272 with 10 cells in Tables 1 and 2. Within one table, the accuracy of MAP estimation with ternary genotype

273 data tends to be higher than that of binary genotype data when fixing other parameters. With the same type

$\mathbf{2 7 4}$ of genotype and same error probability setting, the accuracy decreases as the percentage of missing values

275 increases. When $\alpha$ (or $\beta$ ) is fixed, accuracy tends to decrease as $\beta$ (or $\alpha$ ) increases. Comparing Tables 1 and

2762 , the accuracy of MAP estimation in Table 1 (each tree has 10 cells and longer branch lengths) tends to

$\mathbf{2 7 7}$ be slightly lower than that in Table 2 (each tree has 10 cells and shorter branch lengths) within the same

278 setting with a few exceptions for large $\alpha$ and $\beta$, although the difference is very small. Comparing Tables 3

279 and 4, the effects of branch lengths are flipped and the accuracy of MAP estimation in Table 3 tends to be

280 higher than that in Table 4. 
The results for order accuracy (Tables 5 to 8 in the Supplementary Material) and adjacent order accuracy

282

(Tables 9 to 12 in the Supplementary Material) are similar. In addition to the same overall trends due to number of cells, data type, percentage of missing data and error probabilities, the magnitudes of the order accuracies are higher than the corresponding adjacent order accuracies.

The results for location accuracy, order accuracy and adjacent order accuracy of MO in scenarios 5 to 10 have similar patterns to those observed for scenarios 1 to 4 . The accuracy in scenarios 5 to 10 is not affected by the number of mutations. In addition to the same overall trends due to the number of cells, type of genotype, missing data percentage and error probabilities, the magnitudes of the accuracies in scenarios 5 to 10 are higher than the corresponding accuracies in scenarios 1 to 4 . Especially when error probabilities are low, the accuracies can be as high as $99 \%$.

\subsection{Credible set accuracy}

The credible set accuracy of the inferred mutation branch is assessed as well. If the true mutation branch is within the credible set, we count this as correct; otherwise, it is incorrect. We use $95 \%$ credible set for computation (Tables 13 to 16 in the Supplementary Material). The credible set accuracies have the same overall trends as the accuracies of MAP estimates due to the number of cells, type of genotype, missing data percentage and error probabilities, though the accuracies are much higher than for the corresponding MAP estimates, especially for settings with large error probabilities and higher missing data percentages. As for the MAP estimates, the overall trends for scenarios 5 to 10 are similar to scenarios 1 to 4 , but the corresponding magnitudes of the accuracies in scenarios 5 to 10 are higher than those in scenarios 1 to 4 .

\subsection{Comparison with competing approaches}

To further assess the performance of MO, we compare its performance with the methods SCITE (Jahn and others, 2016) and SiFit (Zafar and others, 2017) for the simulation data in scenarios 1 to 12 . SCITE can estimate the order of mutations for either binary or ternary genotype data. We use the maximum likelihood mutation order inferred by SCITE with 1,000,000 iterations given the true error probabilities. SiFit can use either binary or ternary genotype data when inferring the phylogenetic tree, but it can only use binary genotype data when inferring mutation order. We estimate the most likely mutational profiles for the tips, the internal nodes, and the mutation locations by SiFit given the true phylogenetic tree, error probabilities and mutation rates. We then extract the mutation order information from the output. The three methods are 
309 compared with respect to the order accuracy and adjacent order accuracy for the above simulation settings.

310 3.3.1 Scenarios 1 to 4 Fig. 4 to Fig. 7 plot the order accuracy and the adjacent order accuracy for the

311 three methods for scenarios 1 to 4 , respectively. In each figure, the top row shows the results for binary data

312 and the bottom row shows the results for ternary data. In each panel, different methods are highlighted with

313 different colors.

314 In scenarios 1 to 4, order accuracy and adjacent order accuracy show general decreasing trends as data

315 quality becomes worse for all three methods. For results estimated from the trees with 10 cells (scenarios 1

316 and 2), MO is comparable to SCITE in terms of order accuracy estimated from binary and ternary data.

317 Only when both $\alpha$ and $\beta$ are large does SCITE have higher order accuracy rates than MO. Comparing

318 adjacent order accuracy when there are 10 cells in each tree, MO has comparable adjacent order accuracy

319 when estimated from ternary data. MO has lower adjacent order accuracy than SCITE when estimated from

320 binary data but the discrepancies of adjacent order accuracies between MO and SCITE are only $7 \%$ on

321 average. When there are 50 cells in each tree (scenarios 3 and 4), MO is superior to SCITE in all settings

322 in terms of order accuracy and adjacent order accuracy estimated from both binary and ternary genotype

323

324

331 cells increases. Although the number of correct pairs inferred by SCITE increases, the accuracy decreases

332 because the total number of true pairs increases.

333 3.3.2 Scenarios 5 to 8 Fig. S2 and Fig. S3 in the Supplementary Material plot the order accuracy and

334 adjacent order accuracy for scenarios 5 and 6 , respectively. In scenarios 5 and 6 where mutations evolve by

335 the infinite sites diploid model, order accuracy and adjacent order accuracy show general decreasing trends

336 as data quality becomes worse for all three methods, as is observed for scenarios 1 to 4 . MO is superior to

337 SCITE in all settings in terms of adjacent order accuracy and order accuracy for both the complete and 
338 missing data settings. In all the settings, SiFit has the worst performance with respect to order accuracy

339

340

341

342

$343 \mathrm{MO}$ and SiFit very much. However, the performance of SCITE is affected by the number of mutations. As

344 the number of mutations increases, the accuracy of SCITE becomes worse. In addition, the adjacent order

345

347 for scenarios 7 and 8, respectively. In scenarios 7 and 8, mutations arise by the infinite sites diploid model,

348 accuracy of SCITE increases as the number of cells increases.

Fig. S4 and Fig. S5 in the Supplementary Material plot the order accuracy and the adjacent order accuracy as was the case for scenarios 5 and 6 , but now a small proportion of the mutations are lost. Compared to the complete settings in scenarios 5 and 6 , the performance of all the three methods becomes worse. However, the performance of the three methods is comparable to settings with missing values in scenarios 5 and 6 .

In addition to the above comparisons, we also apply MO to data from scenarios 5 and 6 when transition rates are misspecified. Fig. S9 and Fig. S10 show the order accuracy and adjacent order accuracy when MO is applied with misspecified transition rates $\lambda_{1}=1$ and $\lambda_{2}=10^{5}$. In each panel, red, blue, and green correspond to MO, SCITE, and SiFit, respectively, when the true transition rates $\left(\lambda_{1}=1\right.$ and $\left.\lambda_{2}=0\right)$ are used, as in the initial analysis in scenarios 5 and 6 . Purple color corresponds to MO when the misspecified transition rates are used. The performance of SCITE is not affected by misspecified transition rates. Comparing the plots, we see that when binary data are used, the effect of misspecified transition rates are ignorable, and the accuracy with either the correct or the incorrect transition rates are nearly identical. However, when using ternary data, the differences are noticeable. In scenario 5, the order accuracy for MO with misspecified transition rates is comparable to SCITE when error probabilities are small and higher than SCITE when error probabilities are large. In scenario 6, the order accuracy inferred from ternary genotype data for MO with misspecified transition rates is lower than SCITE. Comparing the adjacent order accuracy with ternary data, the performance of MO with the misspecified transition rates is worse than when the transition rates 
3653.3 .3 Scenarios 9 to 10 In scenarios 9 and 10, mutations are simulated under the mutation process

366

367

368

369

370

371

372

defined in Section 2.2. Although the transition rates are the same as in scenarios 1 to 4 , each mutation is not equally likely to occur on all of the branches. In Fig. S6 and Fig. S7, we observe that MO has higher accuracy than SCITE and SiFit in all settings in terms of both order accuracy and adjacent order accuracy.

3.3.4 Scenarios 11 to 12 In scenarios 11 and 12, mutations are simulated under the finite sites assumption. Because it is unclear how mutation order should be defined when mutations can arise multiple times along a phylogeny, we instead plot the location accuracy of MO and SiFit in Fig. S8. When there are only 10 tips in the tree, most simulated mutations occur only once along the tree and MO has higher accuracy than SiFit. However, when there are 50 tips, most are back mutations and/or parallel mutations. SiFit performs better than $\mathrm{MO}$ when the data are complete and the missing percentage is low. When the missing percentage is high (e.g., 20\%), neither MO nor SiFit identify the correct mutation location. MO is limited by its assumption that all mutations occur only once on the tree. Although SiFit can infer parallel/back mutations, it is not able to identify all the locations on which the mutations occur for the simulated data.

\section{EMPIRICAL EXAMPLES}

We apply MO to two experimental single-cell DNA sequencing datasets, one for prostate cancer (Su and others, 2018) and one for metastatic colon cancer patients (Leung and others, 2017). For the prostate cancer dataset, we retrieve publicly available data from the single-cell study of Su and others (2018), which includes 10 single-cell genomes for each patient. For the colon cancer dataset, we use the somatic single nucleotide variants (SNVs) after variant calling provided in the original study (16 SNVs for patient CRC1 and 36 SNVs for patient CRC2) of Leung and others (2017).

\subsection{Prostate cancer data}

4.1.1 Data analysis To infer tumor evolutionary trees for patients 1 and 2 (labeled P1 and P2), we use the SVDQuartets method of Chifman and Kubatko (2014) as implemented in PAUP* (Swofford, 1999) using the aligned DNA sequences for all somatic mutations as input with the expected rank of the flattening matrix set to 4 . We specify the normal cell sample as the outgroup. We use the maximum likelihood method to estimate the branch lengths.

We select common tumor suppressor genes and oncogenes for both P1 and P2 identified by Su and others (2018). In addition to these common cancer-associated genes across different cancers, we map mutations in 
prostate cancer-specific genes (genes that are more commonly mutated in prostate cancer patients) suggested phylogeny for each of the two patients. $\mathrm{Su}$ and others $(2018)$ estimated the error probabilities to be $(\alpha, \beta)=$

397

414 the Supplementary Material.

415 4.1.2 Results Fig. 8 and Fig. 9 show the tumor evolutionary tree estimated for P1 and P2, respectively.

417 inferred mutation branches. The uncertainty in the inferred mutation locations is highlighted with colors.

418 Mutations with strong signal (defined to be a probability larger than 0.7 that the mutation occurred on a 419 single branch) are colored red, while mutations with moderate signal (defined to be a total probability larger 420 than 0.7 on two or three branches) are colored blue. 
421

422

423

424

425

431

441

We also compare the estimated posterior probability distributions for each mutation of common cancerassociated genes for patients $\mathrm{P} 1$ and $\mathrm{P} 2$, which are used to construct credible sets and to measure the uncertainty of the inferred mutation order. Fig. S11 to Fig. S14 in the Supplementary Material are heatmaps for the posterior probability distribution of each mutation for patients P1 and P2 with different priors (larger or smaller variance).

Fig. 12 shows heatmaps of the estimated posterior probabilities for prostate cancer-specific genes when the variance of the prior distributions are large for P1 and P2. The corresponding heatmaps for the cases in which the prior distribution has small variance are shown in Fig. S15 and Fig. S16 in the Supplementary Material. In agreement with the results of Su et al. (2018), we find that TP53, a mutation commonly associated with tumor initiation in many cancers (see, e.g., Yu and others (2014)), is inferred to occur on the trunk of the tree with high probability in patient P1, but not in patient P2. Gene ZFHX3 has a high probability of having mutated on the trunk of the tree in both patients. In addition, the data for patient P1 shows strong signal that FOXP1 mutates on the trunk of the tumor tree, while BRCA2 has a high probability of having mutated on the trunk of the tree for patient P2. Comparing the heatmaps of common cancer-associated genes with the prostate cancer-specific genes, mutations inferred to have occurred on the trunk of the tree tend to be those that are common across cancer types, while mutations known to have high frequency within prostate cancer are generally found closer to the tips of the tree in both patients.

\subsection{Metastatic colorectal cancer data}

4.2.1 Data analysis The SVDQuartets method of Chifman and Kubatko (2014) is also applied to these data to estimate each colorectal patient's tumor phylogeny. The normal cells in each patient are merged into one normal sample and used as the outgroup. We also merge collections of cells with high similarity (similar mutations). We use the maximum parsimony method to compute the number of changes on each branch and scale the number of changes on each branch by the total number of changes to estimate the branch lengths.

The original study of Leung and others (2017) reported 16 and 36 SNVs for patients CRC1 and CRC2 after variant calling. Leung and others (2017) reported error probabilities of $(\alpha, \beta)=(0 \cdot 0152,0 \cdot 0789)$ and $(\alpha, \beta)=(0 \cdot 0174,0 \cdot 1256)$ for $\mathrm{CRC} 1$ and $\mathrm{CRC} 2$, respectively. For each patient, we use these values to specify

447 the same priors across all sites. For CRC1, we consider $\alpha \mid \mathbf{S}_{i} \sim \operatorname{Beta}(0 \cdot 015,0.985)$ (larger variance) and $\alpha \mid \mathbf{S}_{i} \sim \operatorname{Beta}(0 \cdot 15,9 \cdot 85)$ (smaller variance); and $\beta \mid \mathbf{s}_{i} \sim \operatorname{Beta}(0.078,0.922)$ (larger variance) and $\beta \mid \mathbf{S}_{i} \sim$ 
$\operatorname{Beta}(0 \cdot 78,9 \cdot 22)$ (smaller variance). For CRC2, we consider $\alpha \mid \mathbf{S}_{i} \sim \operatorname{Beta}(0 \cdot 0174,0 \cdot 9826)$ (larger variance)

450 and $\alpha \mid \mathbf{S}_{i} \sim \operatorname{Beta}(0 \cdot 174,9 \cdot 826)$ (smaller variance); and $\beta \mid \mathbf{S}_{i} \sim \operatorname{Beta}(0 \cdot 1256,0 \cdot 8744)$ (larger variance) and $\beta \mid \mathbf{S}_{i} \sim \operatorname{Beta}(1 \cdot 256,8 \cdot 744)$ (smaller variance). The priors for the transition rates for CRC1 and CRC2 are same as for P1 and P2. As was found for the prostate cancer patients, the estimated probabilities do not vary substantially when we use priors with small or large variance.

4.2.2 Results The inferred tumor tree and mutation order are depicted in Fig. 10 and Fig. 11. The posterior probabilities of the inferred mutation locations are indicated with colors as for the prostate cancer data, and agree overall with the findings of Leung and others (2017). Fig. S17 and Fig. S18 in the Supplementary Material are heatmaps for the posterior probability distribution of each mutation for patients CRC1 and $\mathrm{CRC} 2$ with different priors. For patient CRC1, mutations in APC, KRAS and TP53 are inferred to have been acquired on the trunk of the tumor phylogeny with high posterior probability, in agreement with Leung and others (2017) and in agreement with past studies. The studies of Fearon and Vogelstein (1990) and Powell and others (1992) have shown that the mutation order of these genes appears to be fixed in initializing colorectal cancer, providing further support for our findings. In addition, we identify the five mutations specific to metastatic cells that are found by Leung and others (2017), with three (ZNF521, TRRAP, EYS) inferred to occur on branch 21 in Fig. 10 and the remaining two (RBFOX1, GATA1) inferred to occur on branch 29. For CRC2, we identify strong signals on branch 2 in Fig. 11 for 7 genes reported by Leung and others (2017) that are shared by primary and metastatic cells, including driver mutations in APC, NRAS and TP53. We also identify an independent lineage of primary diploid cells (colored in pink in Fig. 11) that evolved in parallel with the rest of the tumor with moderate to strong signals for mutations in ALK, ATR, EPHB6, SPEN and NR3C2 and that do not share the mutations listed in the previous sentence. Our analysis further agrees with that of Leung and others (2017) in that we also identify the subsequent formation of independent metastatic lineages. For example, on branch 124 we find strong support for mutations in FUS; on branch 125 we find strong support for mutations in $A T P 7 B$ and $N R 4 A 3$; and on branch 133 mutations in $H E L Z$ and PRKCB are strongly supported. Many of the genes showing weaker or moderate support for mutation

74 in these metastatic lineages agree with those identified by Leung and others (2017). The primary difference 5 between our result and that of Leung and others (2017) is that we identify mutation in $A T P$ r $B$ along a second major metastatic lineage, rather than in the primary tumor. 


\section{Discussion}

478 Development of computational tools based on a phylogenetic framework for use in studying cancer evolution

479 has the potential to provide tremendous insight into the mechanisms that lead to ITH, especially the role

480 of the temporal order of mutations in cancer progression. For example, Ortmann et al. (2015) have shown

481 differences in clinical features and the response to treatment for patients with different mutation orders,

482 indicating that inference of the order in which mutations arise within an individual's cancer may have direct

483 implications in clinical oncology, both for diagnostic applications in measuring the extent of ITH and for

484 improving targeted therapy. SCS data provide an unprecedented opportunity to estimate mutation order at

485 the highest resolution. However, such data are subject to extensive technical errors that arise during the

486 process of whole-genome amplification.

487

488

489

490

491 to the technical noise that is common for SCS data, including ADO, false positive errors, low-quality data,

492 and missing data. The assumption of independence of mutations made by MO is the same as that made in

493

494 and Jahn and others (2016)). Thus, MO does not presently account for possible interactions between the

495 occurrences of mutations, though it could be extended to accommodate this if biological information about

496 these interactions is available. However, recent work (Canisius and others, 2016) indicates that observed

497 dependence typically takes the form of mutual exclusivity (i.e., only one gene in the group will be mutated

498 in any given patient) rather than positive association, making the independence assumption of less concern

499 here, as the set of mutations we study are assumed to be present within an individual patient. MO could

500 also be extended to work on clonal trees and models that include errors in observed data for multiple cells

501 in a tip instead of a single cell. In addition, MO could be modified to account for the accelerated mutation

502 rates common in late-stage cancers, or to allow for back or parallel mutation.

503 An important difference between MO and existing methods, such as SCITE (Jahn and others, 2016) and

504 SiFit (Zafar and others, 2017), is the mechanism for quantifying uncertainty in the inferred order. Options 
505 available within SCITE (Jahn and others, 2016) allow for estimation of the posterior probability distribution

506 across orders. SiFit (Zafar and others, 2017), on the other hand, could be modified to account for uncertainty

$\mathbf{5 0 7}$ in the orders because the true tumor phylogeny is unknown and must first be estimated. In contrast, because

508 MO uses a probabilistic model for inferring mutation locations along a fixed tree, it is able to provide an

509 estimate of uncertainty in the inferred locations conditioning on the correct tumor phylogeny, thus capturing

510 a source of uncertainty that differs from what SCITE and SiFit provide. MO performs accurately, as is

511 evident from a comprehensive set of simulation studies that take into account different aspects of modern

512 SCS data sets by examining a wide range of error probabilities, fractions of missing data, branch lengths,

513 and numbers of cells in each tree. The simulation studies also demonstrate that MO outperforms the state-

514 of-the-art methods when the number of cells is large and performs comparably to other methods when the

515 number of cells is small. MO is robust to the technical errors that arise during whole-genome amplification.

516 When applied to data from two prostate cancer patients and from two colorectal cancer patients, MO is able

$\mathbf{5 1 7}$ to not only provide insight into the locations of cancer-associated mutations, but also the level of certainty

518 in the locations. However, MO does not provide estimates of transition rates and error probabilities as do

519 SiFit and SCITE, but rather integrates over uncertainty in these parameters.

520 The methodology underlying MO could be enhanced by incorporating models for copy number alterations,

$\mathbf{5 2 1}$ as well as by considering mutations that affect the same allele more than once. As SCS data collection

$\mathbf{5 2 2}$ becomes more advanced, enabling hundreds of cells to be analyzed in parallel at reduced cost and increased

523 throughput, MO is poised to analyze the resulting large-scale data sets to make meaningful inference of

$\mathbf{5 2 4}$ the mutation order during tumor progression for individual patients. MO thus represents an important step

525 forward in understanding the role of mutation order in cancer evolution and as such may have important

526 translational applications for improving cancer diagnosis, treatment, and personalized therapy. If inferred

$\mathbf{5 2 7}$ mutation orders can be associated with clinical outcomes, future research can explore the cause of clinical

$\mathbf{5 2 8}$ outcomes given specific mutation orders with the goal of developing novel, targeted treatments. This will

529 allow clinical providers to make decisions concerning treatment based on the mutation landscapes of patients.

530 Although the current study focuses on cancer, MO can potentially also be applied to single-cell mutation

531 profiles from a wide variety of fields. These applications are expected to provide new insights into our

532 understanding of cancer and other human diseases. 


\section{Software}

534 MO has been implemented in R and is available at https://github.com/lkubatko/MO.

536 Supplementary material is available.

538 The simulation experiments and data analyses were carried out using the ASC Unity Cluster at The Ohio

539 State University, USA. The authors thank two anonymous reviewers for helpful comments on an earlier draft

540 of this manuscript.

$541 \quad$ Conflict of Interest: None

542

543 Ascolani, Gianluca And Liò, Pietro. (2019). Modeling breast cancer progression to bone: how driver 544 mutation order and metabolism matter. BMC Medical Genomics 12(6), 106.

545 Barbieri, Christopher E, Bangma, Chris H, Buartell, Anders, Catto, James WF, Culig, Zoran, Grönberg, Henrik, Luo, Jun, Visakorpi, Tapio and Rubin, Mark A. (2013). The mutational landscape of prostate cancer. European Urology 64(4), 567-576.

Canisius, Sander, Martens, John W. M. And Wessels, Lodewyk F. A. (2016). A novel independence test for somatic alterations in cancer shows that biology drives mutual exclusivity but chance explains most co-occurrence. Genome Biology 17, 261.

Chifman, Julia And Kubatko, Laura. (2014). Quartet inference from SNP data under the coalescent model. Bioinformatics 30(23), 3317-3324.

Fearon, Eric R and Vogelstein, Bert. (1990). A genetic model for colorectal tumorigenesis. cell 61(5), $759-767$.

Ishwaran, Hemant, Blackstone, Eugene H, Apperson-Hansen, Carolyn and Rice, Thomas W. (2009). A novel approach to cancer staging: application to esophageal cancer. Biostatistics 10(4), 603-620. 
557 Iwasa, Yoh, Michor, Franziska And Nowak, Martin A. (2004). Stochastic tunnels in evolutionary 558 dynamics. Genetics 166(3), 1571-1579.

559 Jahn, Katharina, Kuipers, Jack and Beerenwinkel, Niko. (2016). Tree inference for single-cell data. $560 \quad$ Genome Biology $\mathbf{1 7}(1), 86$.

561 Jamal-Hanjani, Mariam, Wilson, Gareth A, McGranahan, Nicholas, Birkbak, Nicolai J, 562 Watkins, Thomas BK, Veeriah, Selvaraju, Shafi, Seema, Johnson, Diana H, Mitter, 563 Richard, Rosenthal, Rachel and others. (2017). Tracking the evolution of non-small-cell lung cancer. 564 New England Journal of Medicine 376(22), 2109-2121.

565 Kim, Kyung In And Simon, Richard. (2014). Using single cell sequencing data to model the evolutionary 566 history of a tumor. BMC Bioinformatics $\mathbf{1 5}(1), 27$.

567 Leung, Marco L., Davis, Alexander, Gao, Ruli, Casasent, Anna, Wang, Yong, Sei, Emi, Vilar, 568 Eduardo, Maru, Dipen, Kopetz, Scott and Navin, Nicholas E. (2017). Single-cell DNA sequencing reveals a late-dissemination model in metastatic colorectal cancer. Genome Research 27, 1287-1299.

570 Navin, Nicholas E. (2014). Cancer genomics: one cell at a time. Genome Biology 15(8), 452.

571 Ortmann, Christina A, Kent, David G, Nangalia, Jyoti, Silber, Yvonne, Wedge, David C, 572 Grinfeld, Jacob, Baxter, E Joanna, Massie, Charles E, Papaemmanuil, Elli, Menon, Suraj 573 and others. (2015). Effect of mutation order on myeloproliferative neoplasms. New England Journal of $\mathbf{5 7 4} \quad$ Medicine 372(7), 601-612.

575 O'Sullivan, Finbarr, Roy, Supratik and Eary, Janet. (2003). A statistical measure of tissue hetero576 geneity with application to 3D PET sarcoma data. Biostatistics 4(3), 433-448.

577 PosadA, David. (2020). CellCoal: coalescent simulation of single-cell sequencing samples. Molecular Biology $\mathbf{5 7 8}$ and Evolution 37(5), 1535-1542.

579 Powell, Steven M, Zilz, Nathan, Beazer-Barclay, Yasmin, Bryan, Tracy M, Hamilton, StanLey R, Thibodeau, Stephen N, Vogelstein, Bert and Kinzler, Kenneth W. (1992). Apc muta581 tions occur early during colorectal tumorigenesis. Nature 359(6392), 235-237. 
582 Su, Fei, Zhang, Wei, Zhang, Dalei, Zhang, Yaqun, Pang, Cheng, Huang, Yingying, Wang, Miao,

583 Cui, Luwei, He, Lei, Zhang, Jinsong and others. (2018). Spatial intratumor genomic heterogeneity within localized prostate cancer revealed by single-nucleus sequencing. European Urology 74(5), 551-559.

585 Swofford, DL. (1999). Phylogenetic analysis using parsimony, PAUP* 4.0, beta version 4.0 b2. Sinauer $586 \quad$ Associates, Boston, Mass.

587 Tate, John G, Bamford, Sally, Jubb, Harry C, Sondka, Zbyslaw, Beare, David M, Bindal, 588 Nidhi, Boutselakis, Harry, Cole, Charlotte G, Creatore, Celestino, Dawson, Elisabeth 589 and others. (2018). COSMIC: the catalogue of somatic mutations in cancer. Nucleic Acids Re590 search 47(D1), D941-D947.

591 Yu, Chang, Yu, Jun, Yao, Xiaotian, Wu, William K. K., Lu, Youyong, Tang, Senwei, Li, 592 Xiangchun, Bao, Li, Li, Xiaoxing, Hou, Yong, Wu, Renhua, Jian, Min, Chen, Ruoyan, Zhang, 593 Fan, Xu, Lixia, Fan, Fan, He, Jun, Liang, Qiaoyi, Wang, Hongyi, Hu, Xueda, He, Minghui, 594 Zhang, Xiang, Zheng, Hancheng, Li, Qibin, Wu, Hanjie, Chen, Yan, Yang, Xu, Zhu, Shida, 595 Xu, Xun, Yang, Huanming, Wang, Jian, Zhang, Xiuging, Sung, Joseph J. Y., Li, Yingrui and 596 others. (2014). Discovery of biclonal origin and a novel oncogene SLC12A5 in colon cancer by single-cell sequencing. Cell Research 24, 701-712.

598 Zafar, Hamim, Navin, Nicholas, Chen, Ken and Nakhleh, Luay. (2019). SiCloneFit: Bayesian infer599 ence of population structure, genotype, and phylogeny of tumor clones from single-cell genome sequencing 600 data. Genome Research 29, 1-13.

601 Zafar, Hamim, Tzen, Anthony, Navin, Nicholas, Chen, Ken and Nakhleh, Luay. (2017). SiFit: 602 inferring tumor trees from single-cell sequencing data under finite-sites models. Genome Biology 18(1), 603 178. 

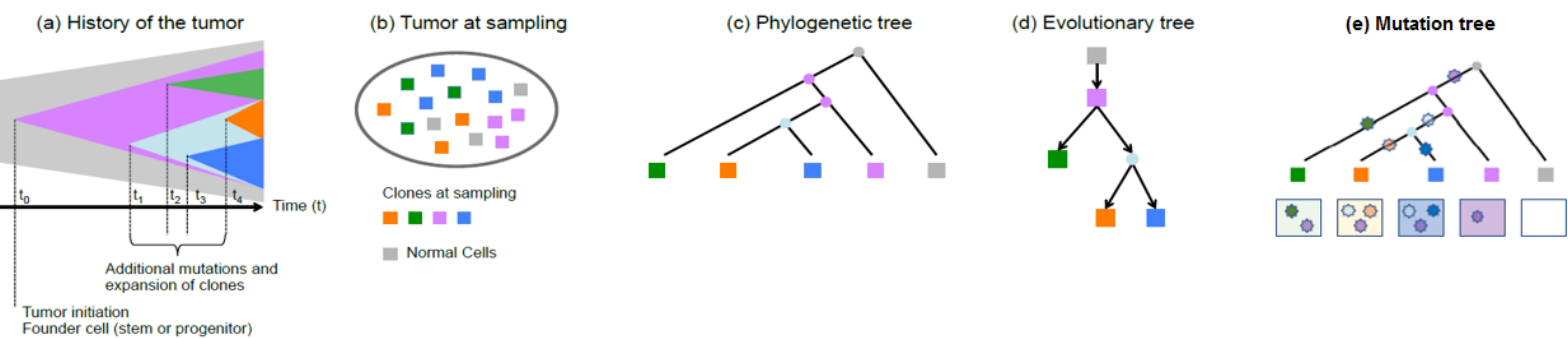

Fig. 1: Pictorial representation of tumor evolution. (a) - (b) A pictorial representation of the evolution of a tumor from the first initiating mutation to the heterogeneous tissue at the time of sampling, which consists of four different clones and normal tissue. (c) A phylogenetic tree with single cells as the tips. (d) A clonal lineage tree inferred from sampled cells where each node represents a subclone (cluster of cells). (e) A mutation tree inferred from sampled cells where each star represents the occurrence of one mutation. Boxes underneath each tip show which mutations are present in the cell represented by the tip.

(a) True binary genotype

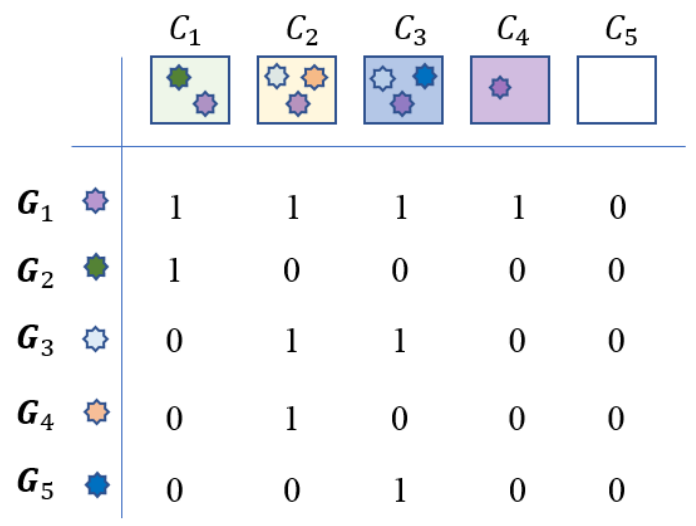

(b) Observed binary genotype

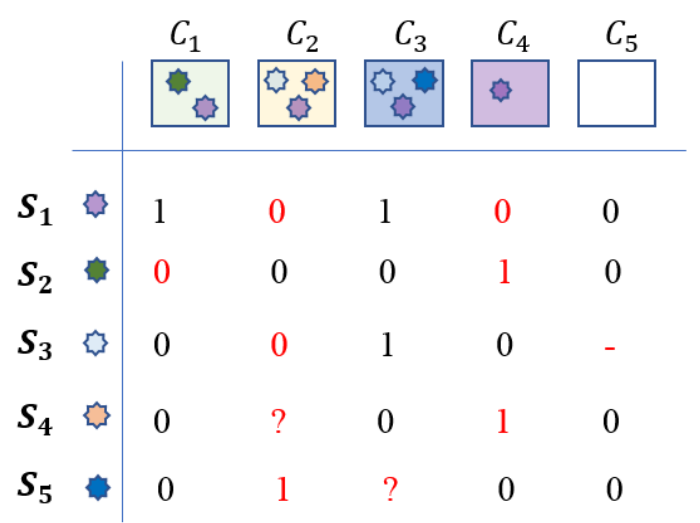

Fig. 2: True and observed binary data. (a) True binary mutation matrix representing the mutation status of the sequenced tumor cells in the mutation tree in Fig. 1(e). Each row represents true genotypes for one genomic site in all cells and each column represents the true genotypes of multiple genomic sites for one single cell. (b) Observed mutation matrix with missing and ambiguous values (red), as well as genotypes that are misrecorded with respect to the true mutation matrix (red numbers; these are either false positives or false negatives). The red dash indicates a missing value since the sequencing process does not return signal at this site of this cell, and the red question mark represents an ambiguous value. Each row represents observed genotypes for one genomic site in all cells and each column represents the observed genotypes of multiple genomic sites for one single cell. 


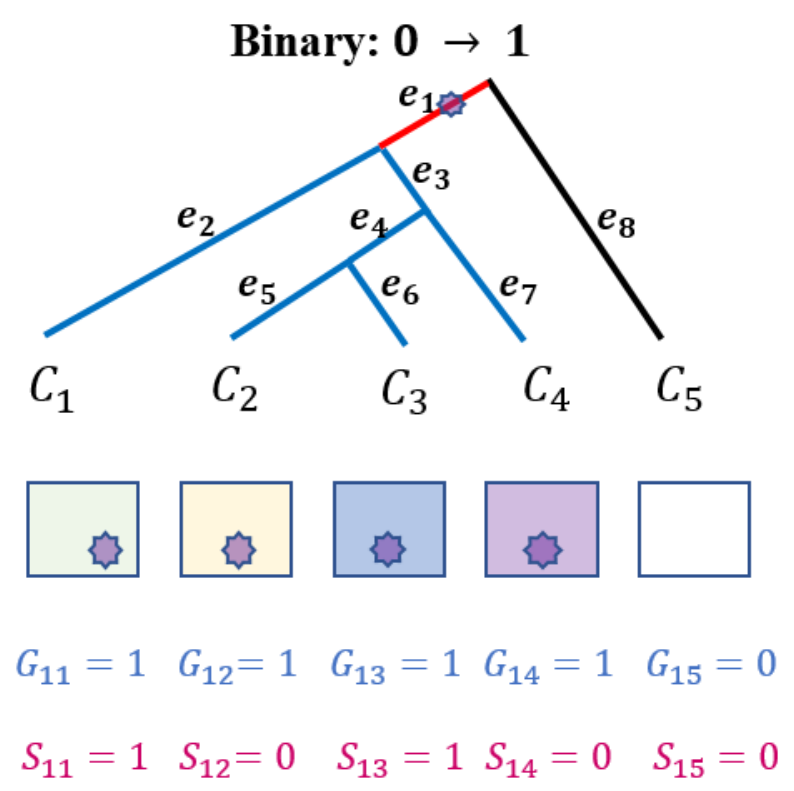

Fig. 3: Binary mutation process example. Example in which a mutation is acquired on branch $e_{1}$ (marked with red color). The cell descending from branch $e_{8}$ (marked with black color) does not carry the mutation, while the cells descending from the blue branches carry the mutation. 
bioRxiv preprint doi: https://doi.org/10.1101/2020.05.06.081398; this version posted January 8, 2021. The copyright holder for this preprint (which was not certified by peer review) is the author/funder, who has granted bioRxiv a license to display the preprint in perpetuity. It is made available under aCC-BY-NC-ND 4.0 International license.
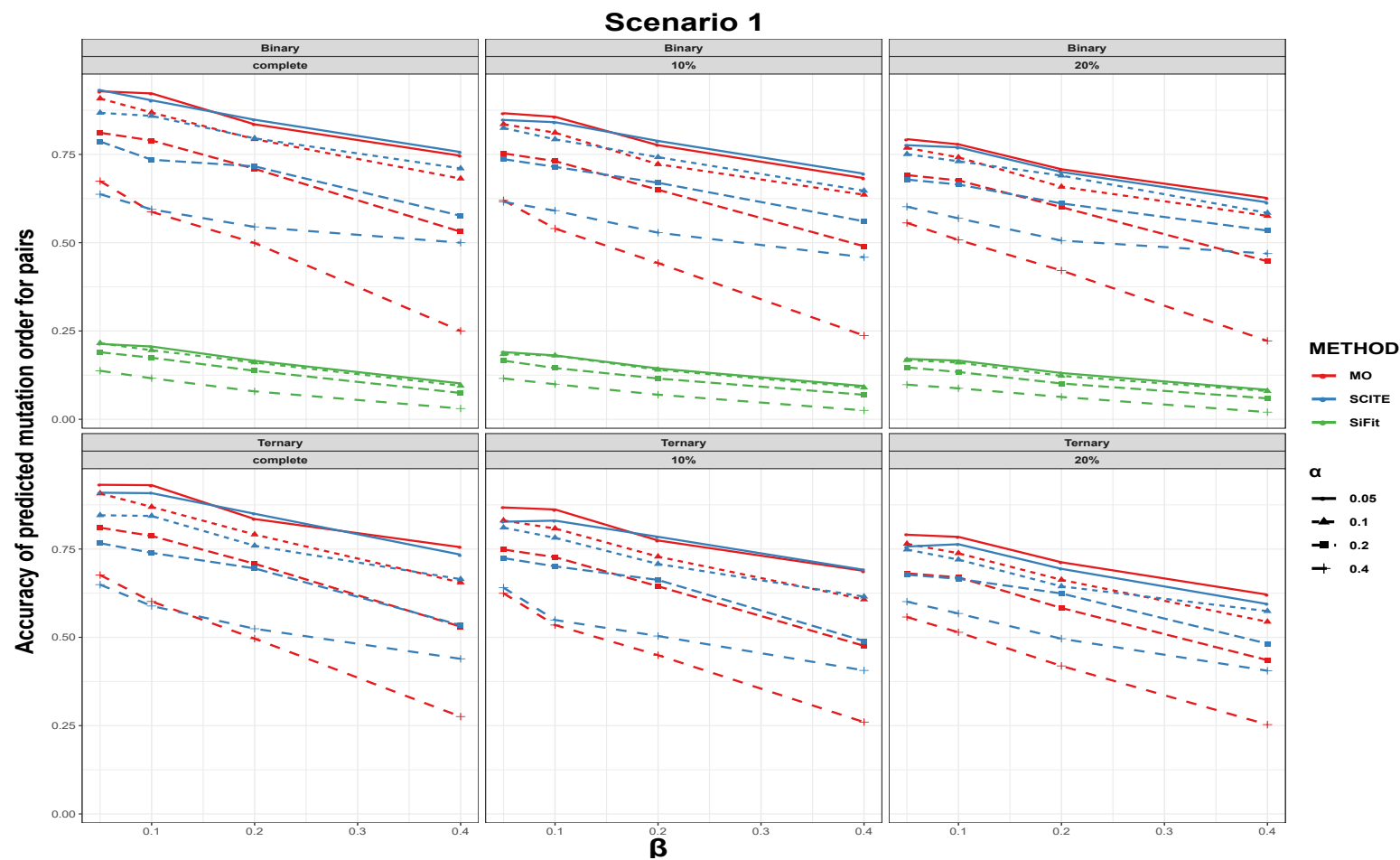

(a)
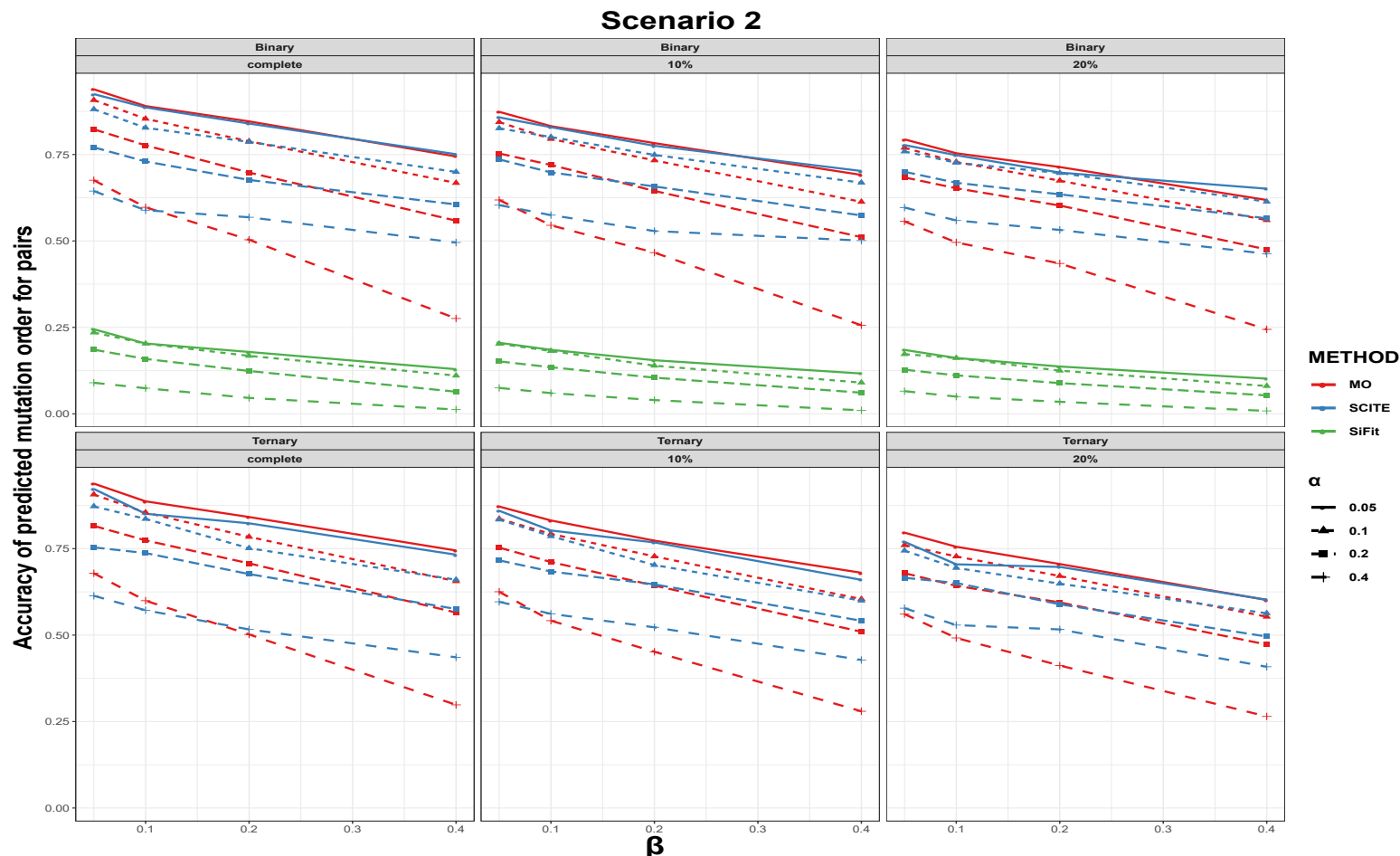

(b)

Fig. 4: Order accuracy in scenarios 1 and 2 for MO, SCITE and SiFit. Each panel includes the results from the specific type of genotype and missing data percentage. In each panel, red, blue and green colors correspond to MO, SCITE and SiFit, respectively. Each plotting symbol on the line represents a different probability of a false positive error, $\alpha$. The $\mathrm{x}$-axis is the probability of a false negative error, $\beta$, and the $\mathrm{y}$-axis is order accuracy. 
bioRxiv preprint doi: https://doi.org/10.1101/2020.05.06.081398; this version posted January 8, 2021. The copyright holder for this preprint (which was not certified by peer review) is the author/funder, who has granted bioRxiv a license to display the preprint in perpetuity. It is made available under aCC-BY-NC-ND 4.0 International license.
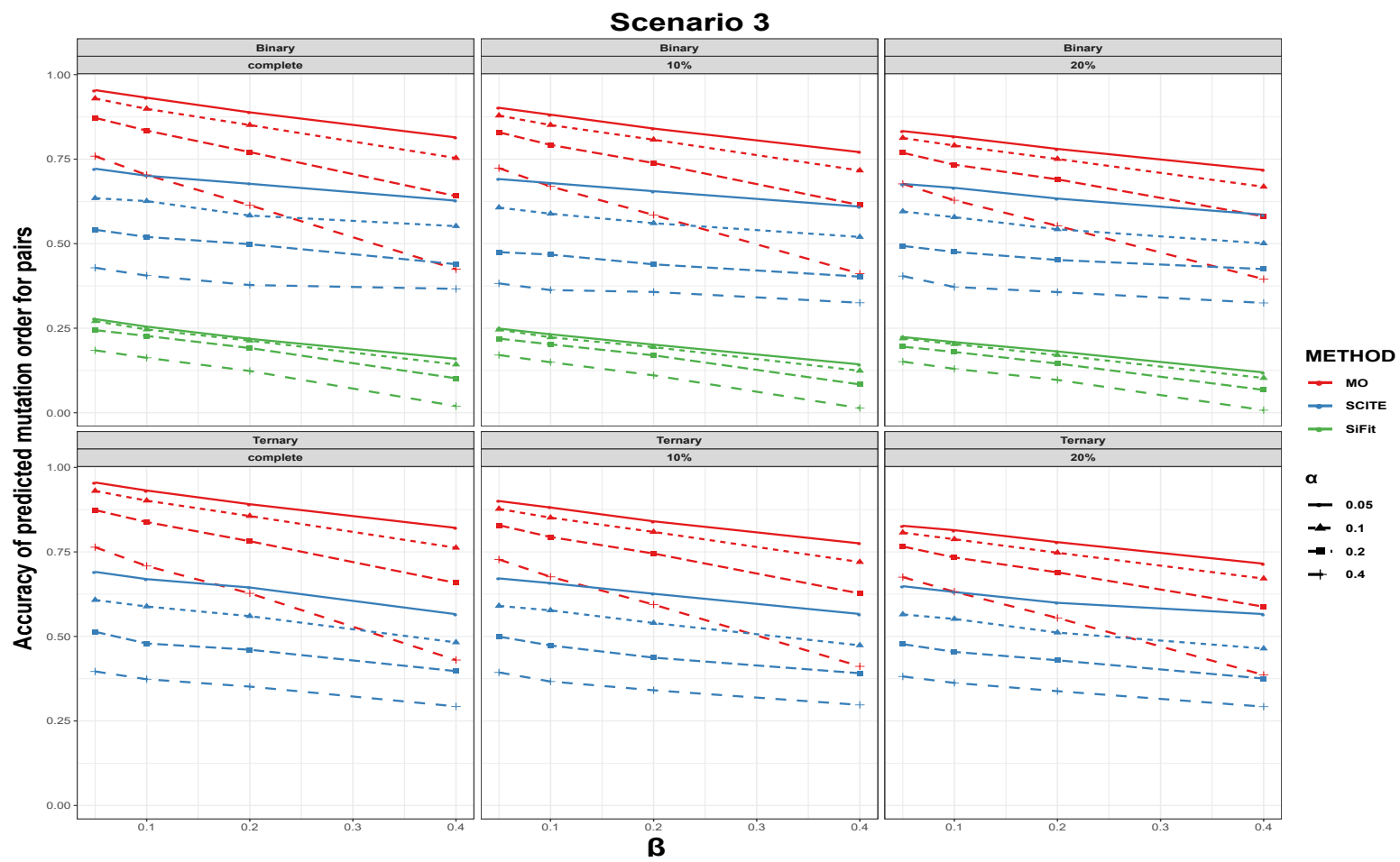

(a)
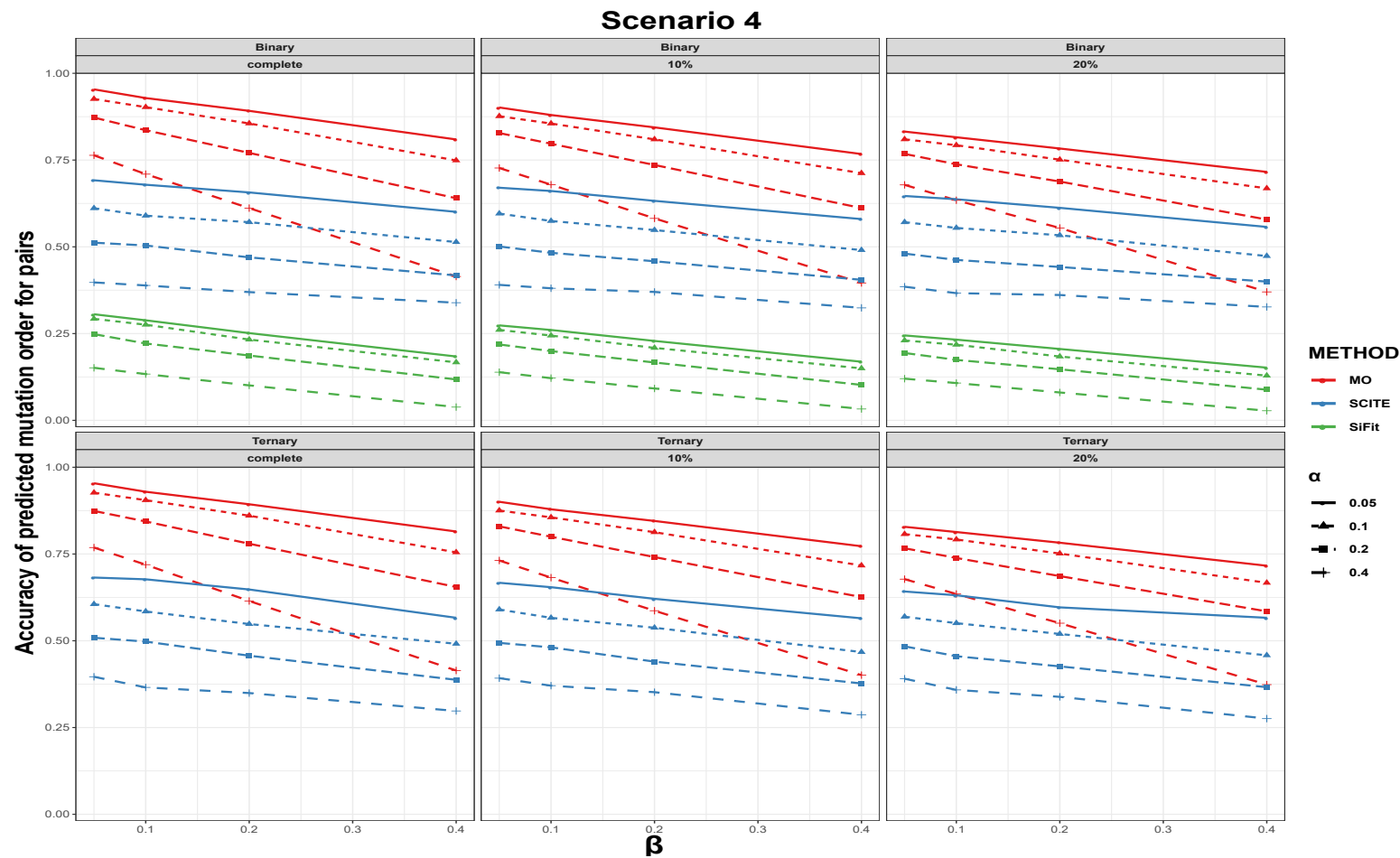

(b)

Fig. 5: Order accuracy in scenarios 3 and 4 for MO, SCITE and SiFit. Each panel includes the results from the specific type of genotype and missing data percentage. In each panel, red, blue and green colors correspond to MO, SCITE and SiFit, respectively. Each plotting symbol on the line represents a different probability of a false positive error, $\alpha$. The $\mathrm{x}$-axis is the probability of a false negative error, $\beta$, and the $\mathrm{y}$-axis is order accuracy. 
bioRxiv preprint doi: https://doi.org/10.1101/2020.05.06.081398; this version posted January 8, 2021. The copyright holder for this preprint (which was not certified by peer review) is the author/funder, who has granted bioRxiv a license to display the preprint in perpetuity. It is made available under aCC-BY-NC-ND 4.0 International license.
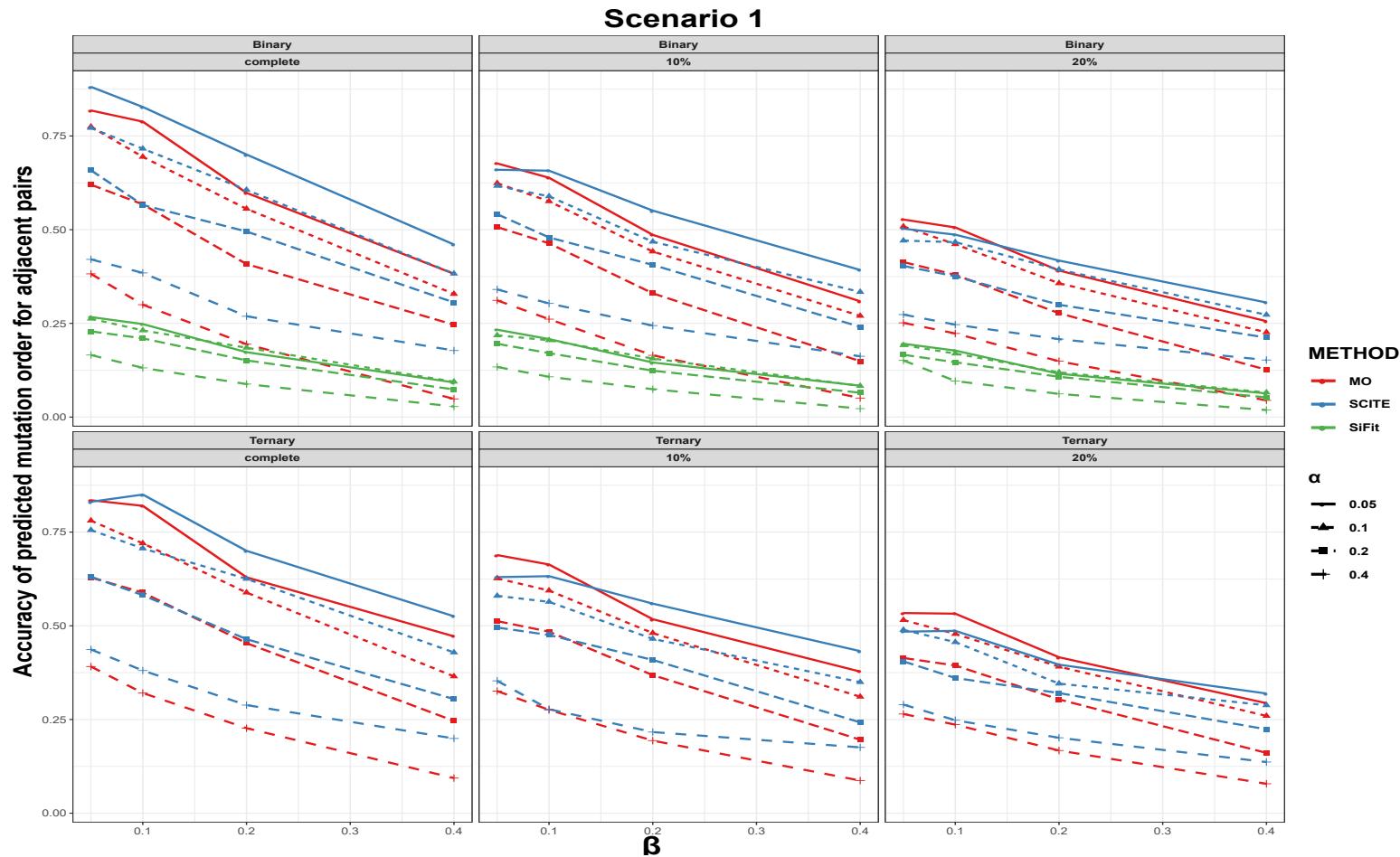

(a)
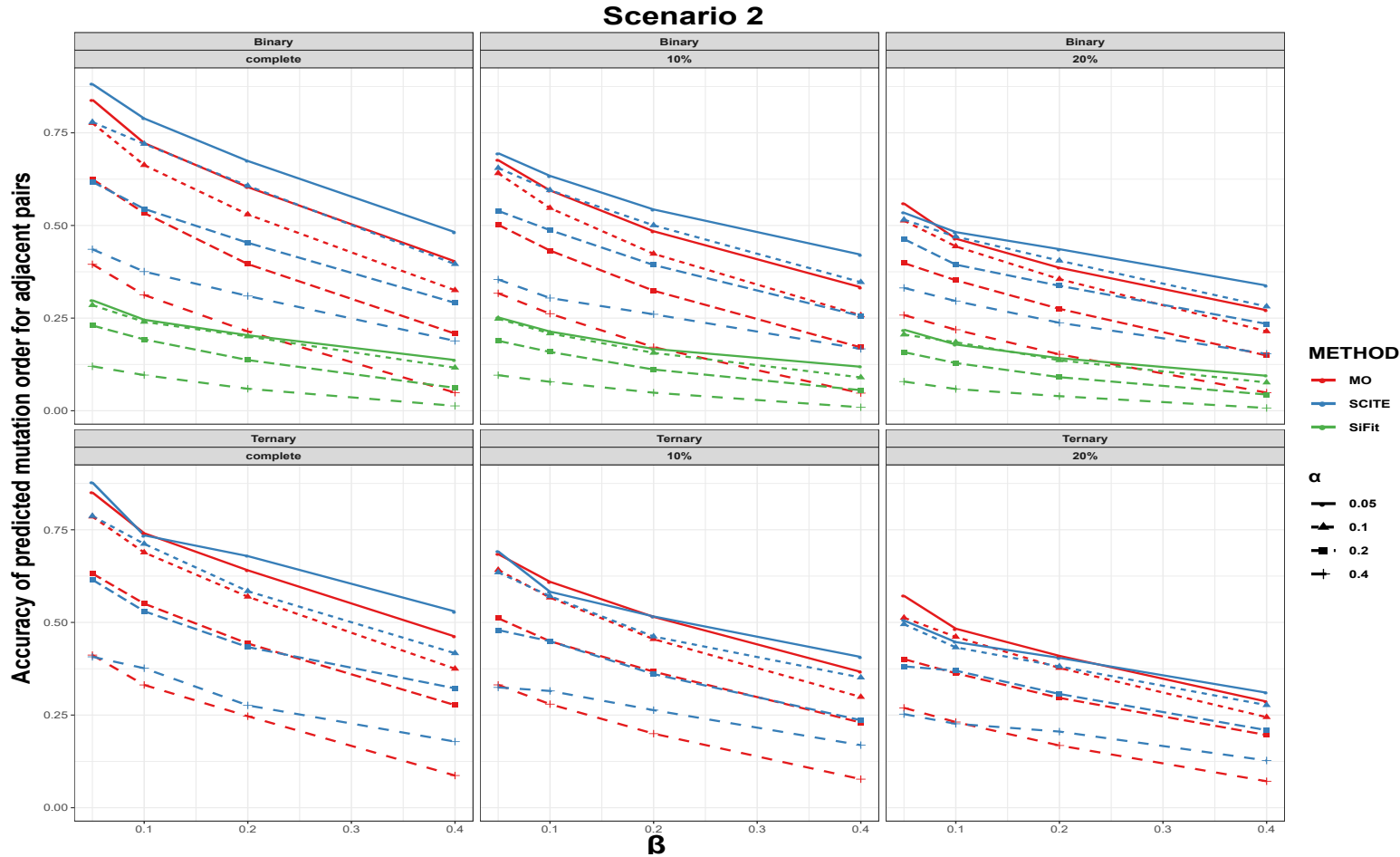

(b)

Fig. 6: Adjacent order accuracy in scenarios 1 and 2 for MO, SCITE and SiFit. Each panel includes the results from the specific type of genotype and missing data percentage. In each panel, red, blue and green colors correspond to MO, SCITE and SiFit, respectively. Each plotting symbol on the line represents a different probability of a false positive error, $\alpha$. The $\mathrm{x}$-axis is the probability of a false negative error, $\beta$, and the $\mathrm{y}$-axis is adjacent order accuracy. 
bioRxiv preprint doi: https://doi.org/10.1101/2020.05.06.081398; this version posted January 8, 2021. The copyright holder for this preprint (which was not certified by peer review) is the author/funder, who has granted bioRxiv a license to display the preprint in perpetuity. It is made available under aCC-BY-NC-ND 4.0 International license.
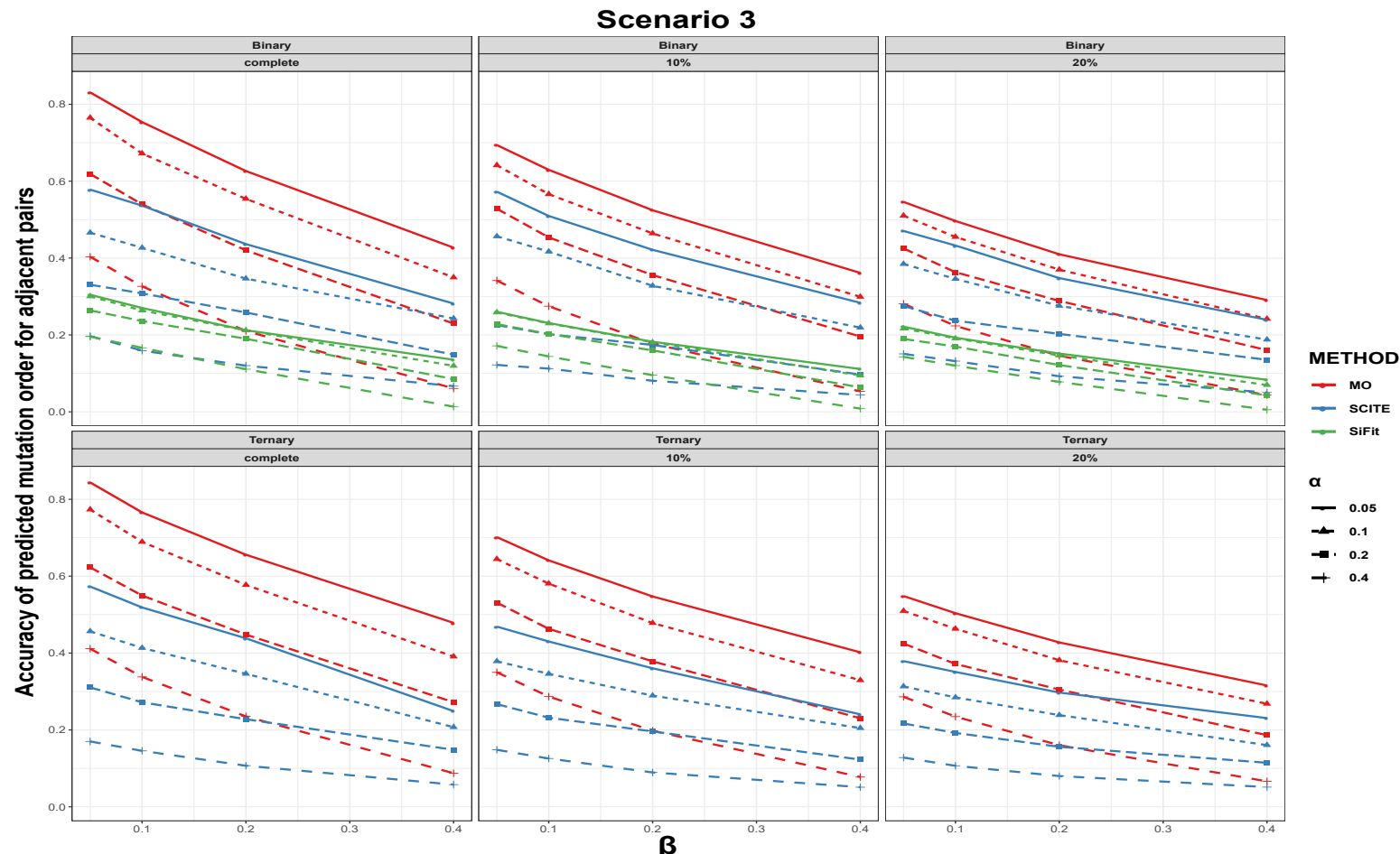

(a)
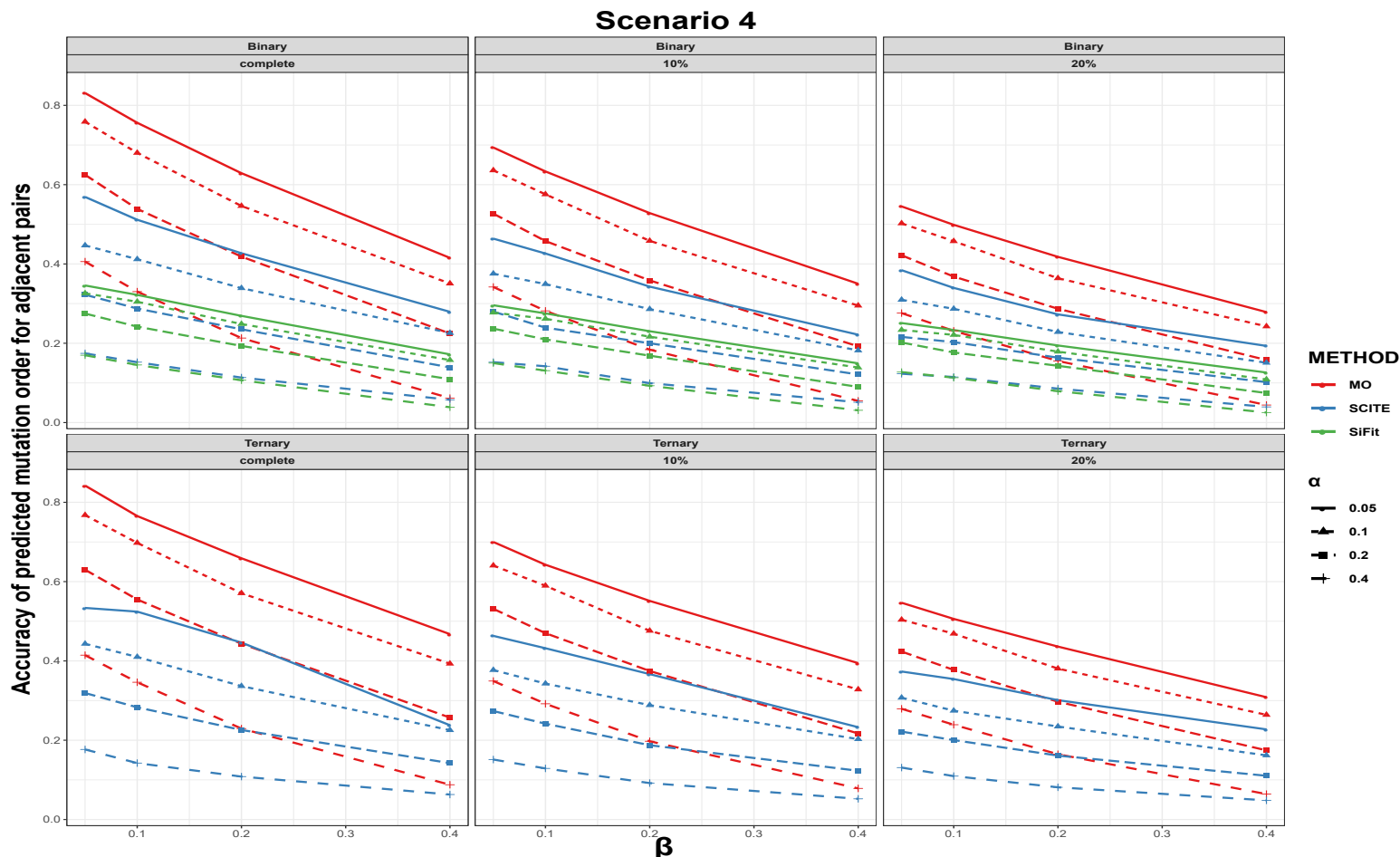

(b)

Fig. 7: Adjacent order accuracy in scenarios 3 and 4 for MO, SCITE and SiFit. Each panel includes the results from the specific type of genotype and missing data percentage. In each panel, red, blue and green colors correspond to MO, SCITE and SiFit, respectively. Each plotting symbol on the line represents a different probability of a false positive error, $\alpha$. The x-axis is the probability of a false negative error, $\beta$, and the $\mathrm{y}$-axis is adjacent order accuracy. 
bioRxiv preprint doi: https://doi.org/10.1101/2020.05.06.081398; this version posted January 8, 2021. The copyright holder for this preprint (which was not certified by peer review) is the author/funder, who has granted bioRxiv a license to display the preprint in perpetuity. It is made available under aCC-BY-NC-ND 4.0 International license.
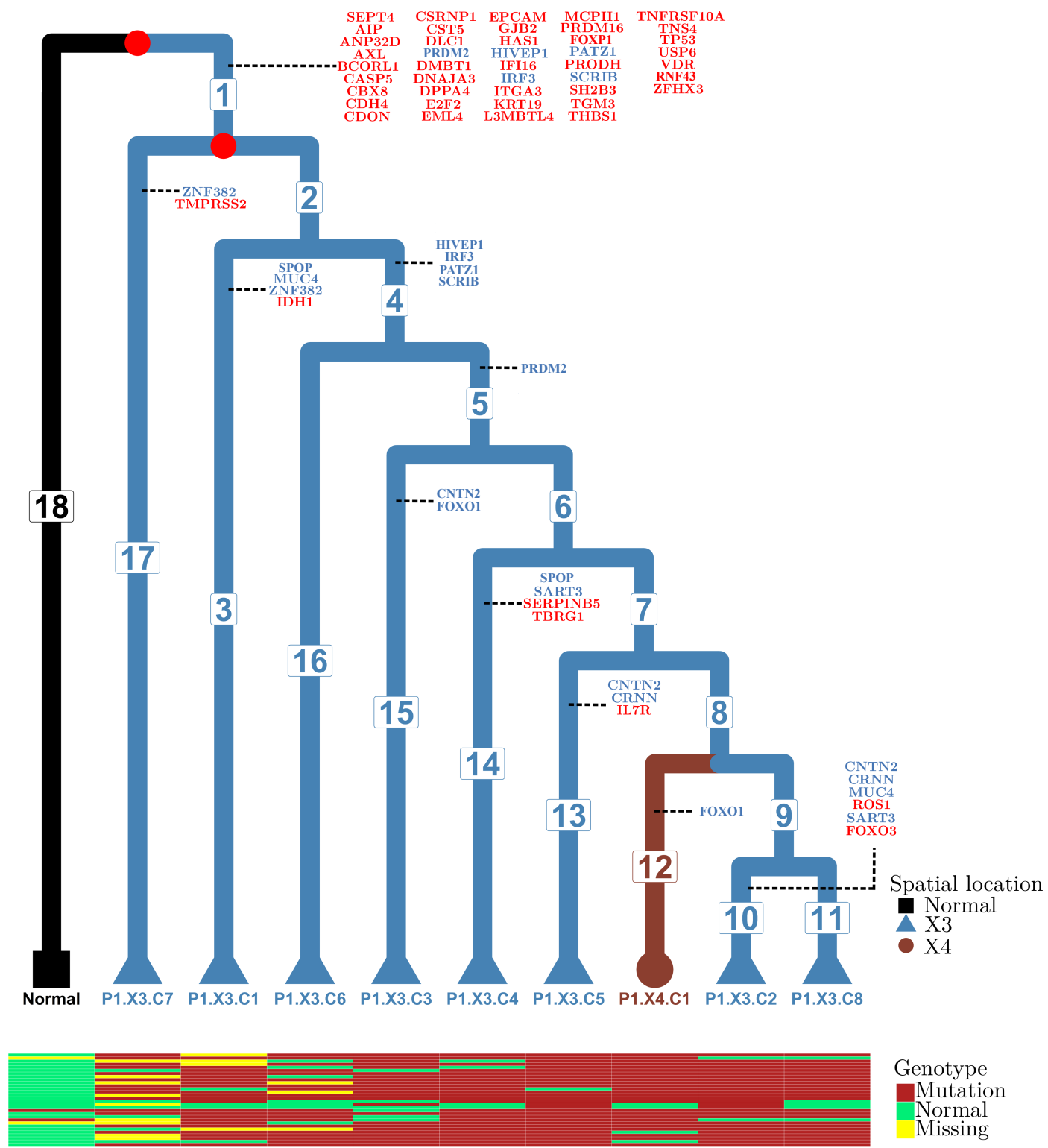

Fig. 8: P1 tumor phylogenetic tree and inferred temporal order of the mutations. The normal cell is set as the outgroup. There are 18 branches in this tree. We do not assume the molecular clock when estimating the branch lengths. Branch lengths in this figure are not drawn to scale. The color and tip shape represent the spatial locations of the samples (normal tissue, location X3 or location X4; see Su and others (2018)). The temporal order of the mutations is annotated on the branches of the tree. The uncertainty of mutation locations is highlighted with colors. Mutations with very strong signals (probability of occurring on one branch is greater than 0.7) are marked in red, while mutations with moderate signals (probabilities that sum to more than 0.7 on two or three branches) are marked in blue. Mutation data for 30 genes corresponding to the first 30 rows in Fig. S11 and Fig. S12 for each tip are shown in the heatmap matrix at the bottom. 
bioRxiv preprint doi: https://doi.org/10.1101/2020.05.06.081398; this version posted January 8, 2021. The copyright holder for this preprint (which was not certified by peer review) is the author/funder, who has granted bioRxiv a license to display the preprint in perpetuity. It is made available under aCC-BY-NC-ND 4.0 International license.

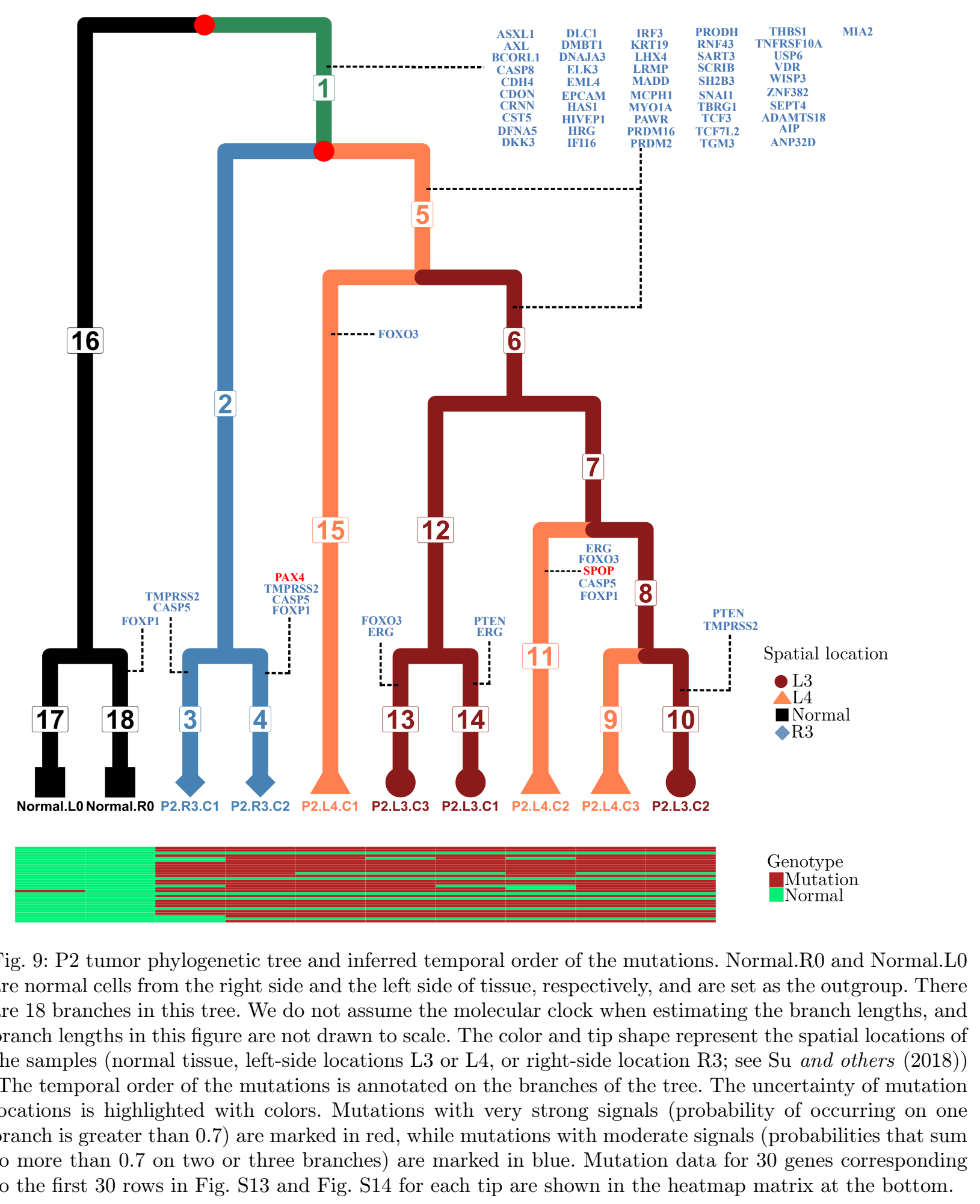


bioRxiv preprint doi: https://doi.org/10.1101/2020.05.06.081398; this version posted January 8, 2021. The copyright holder for this preprint (which was not certified by peer review) is the author/funder, who has granted bioRxiv a license to display the preprint in perpetuity. It is made available under aCC-BY-NC-ND 4.0 International license.
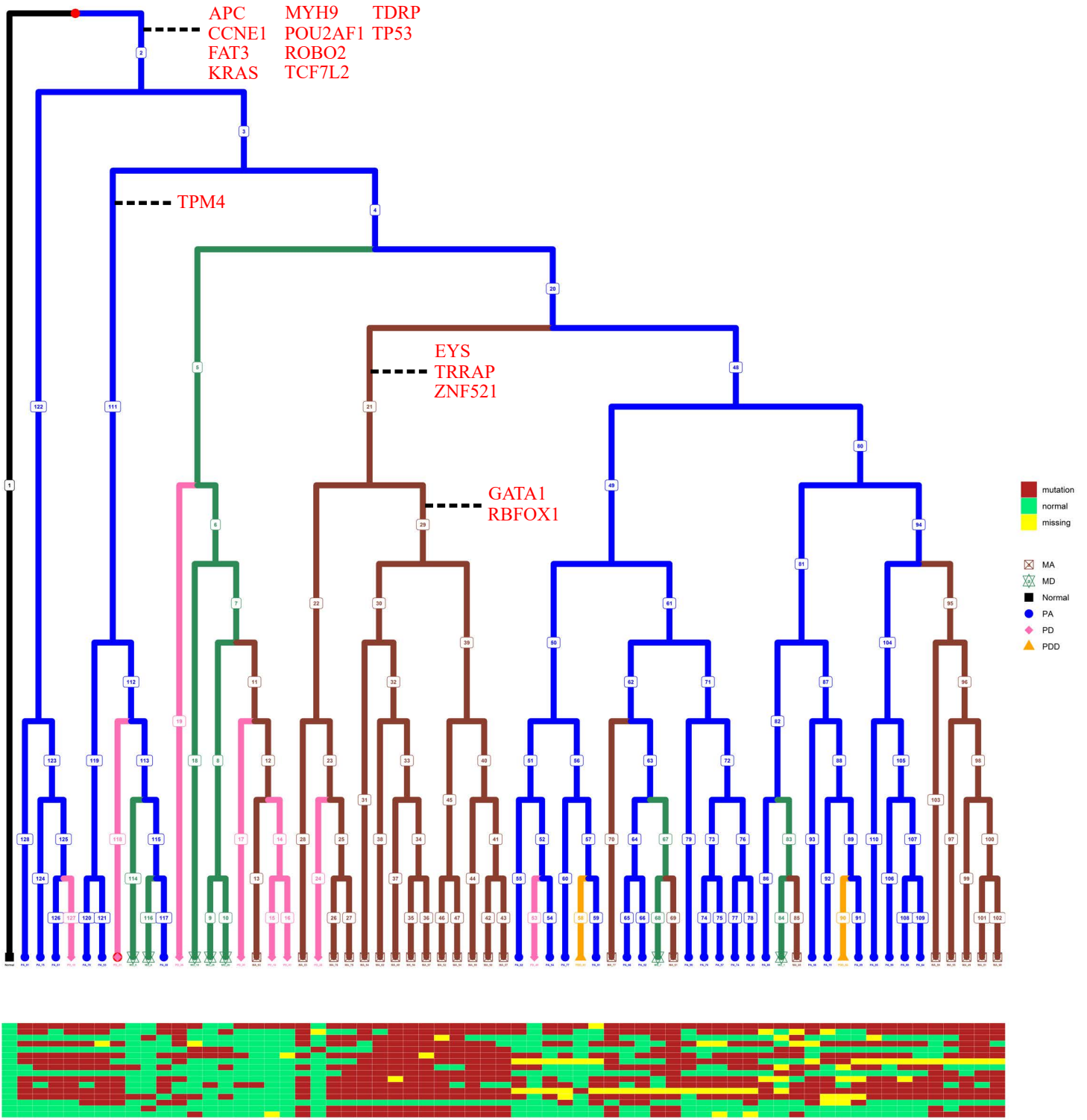

Fig. 10: CRC1 tumor phylogenetic tree and inferred temporal order of the mutations. The color and tip shape represent the spatial locations of the samples (Normal - normal tissue; PA - primary aneuploid; PD - primary diploid; MA - metastatic aneuploid; MD - metastatic diploid; see Leung and others (2017)). The temporal order of the mutations is annotated on the branches of the tree. The uncertainty of mutation locations is highlighted with colors. Mutations with very strong signals (probability of occurring on one branch is greater than 0.7 ) are marked in red, while genes with moderate signals (probabilities that sum to more than 0.7 on two or three branches) are marked in blue. The branch lengths are not scaled. Mutation data for the 16 genes corresponding to each tip are shown in the heatmap matrix at the bottom. 
bioRxiv preprint doi: https://doi.org/10.1101/2020.05.06.081398; this version posted January 8, 2021. The copyright holder for this preprint (which was not certified by peer review) is the author/funder, who has granted bioRxiv a license to display the preprint in perpetuity. It is made available under aCC-BY-NC-ND 4.0 International license.

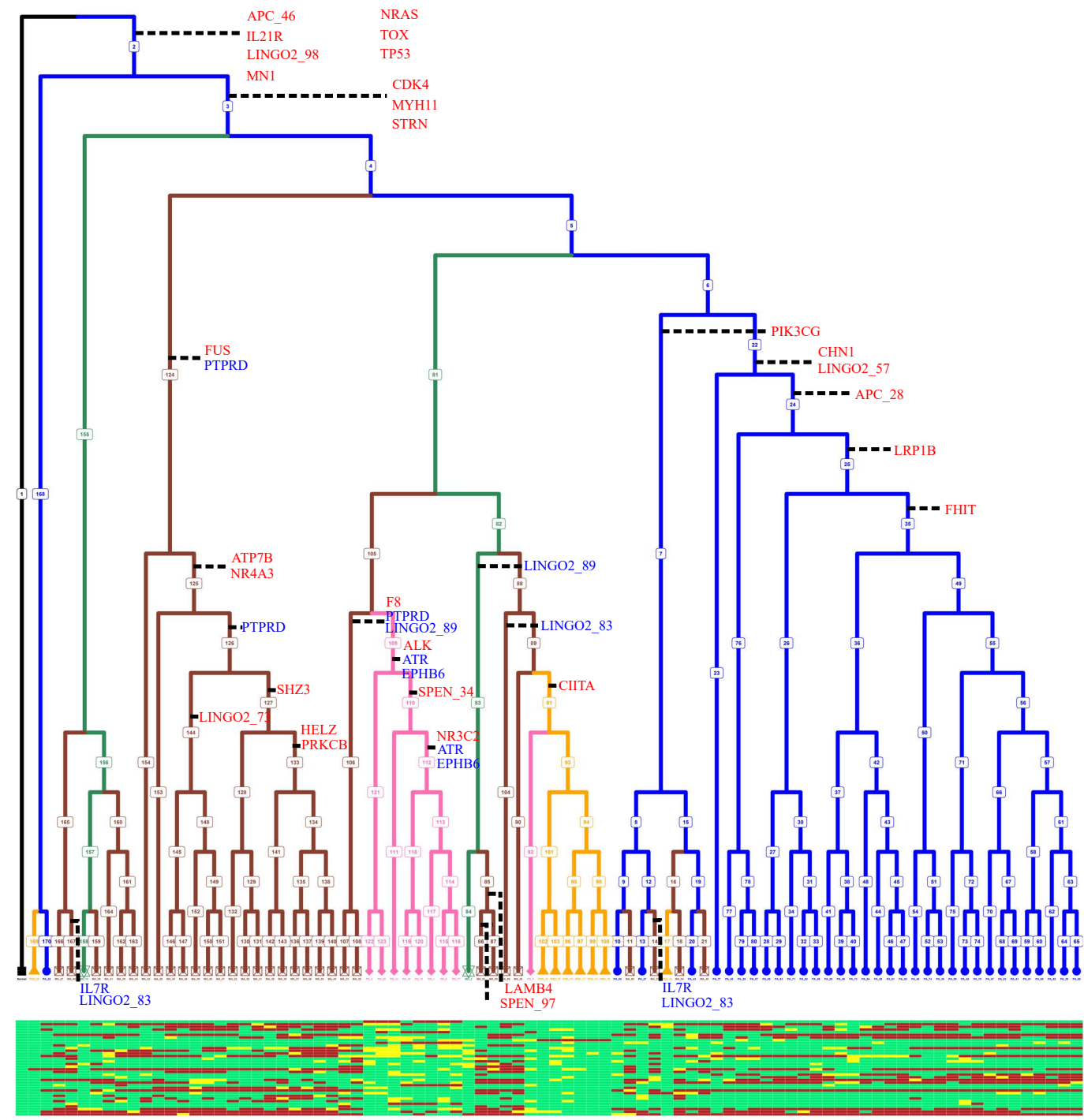

Fig. 11: CRC2 tumor phylogenetic tree and inferred temporal order of the mutations. The color and tip shape represent the spatial locations of the samples (Normal - normal tissue; PA - primary aneuploid; PD - primary diploid; MA - metastatic aneuploid; MD - metastatic diploid; see Leung and others (2017)). The temporal order of the mutations is annotated on the branches of the tree. The uncertainty of mutation locations is highlighted with colors. Mutations with very strong signals (probability of occurring on one branch is greater than 0.7) are marked in red, while mutations with moderate signals (probabilities that sum to more than 0.7 on two or three branches) are marked in blue. The branch lengths are not scaled. Mutation data for the 36 genomic sites corresponding to each tip are shown in the heatmap matrix at the bottom. 
(a) P1 prostate cancer specific mutations with binary data

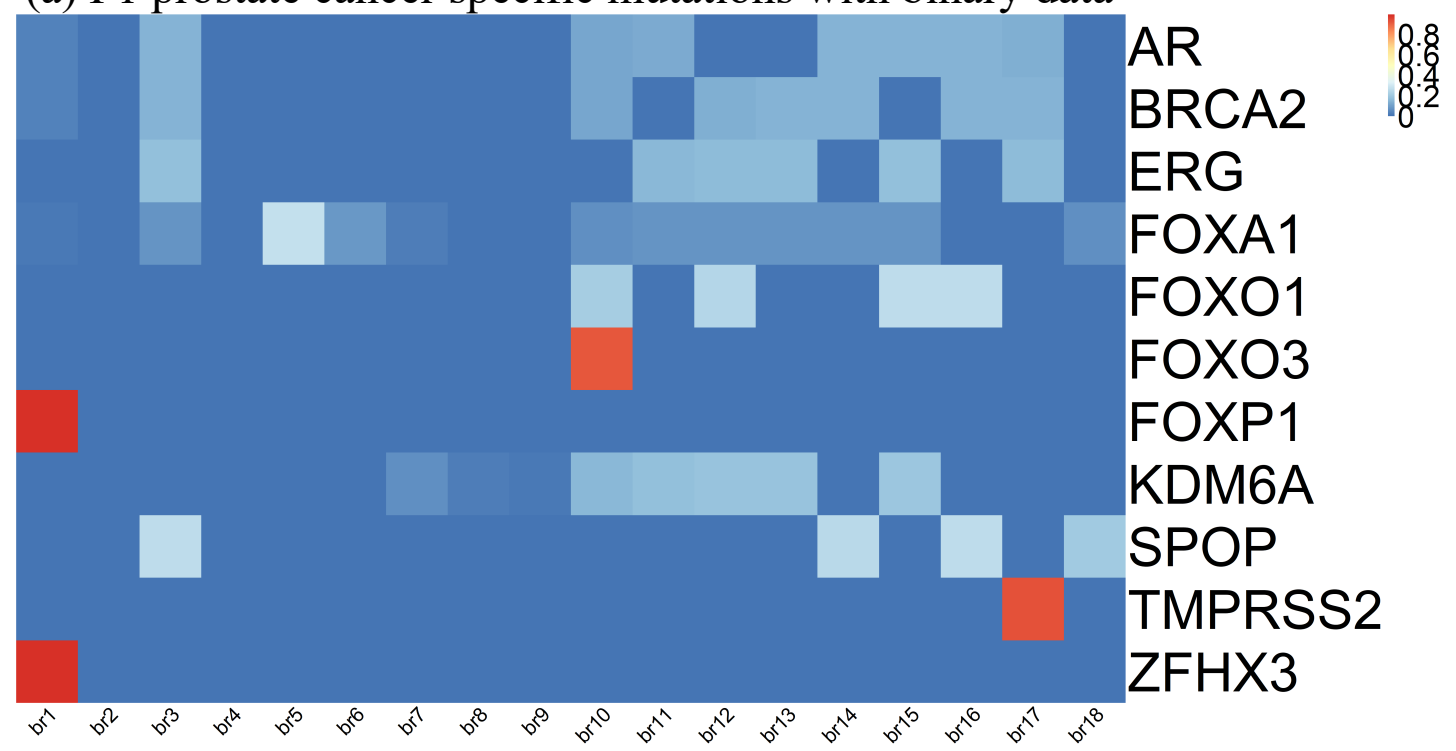

(b) P1 prostate cancer specific mutations with ternary data

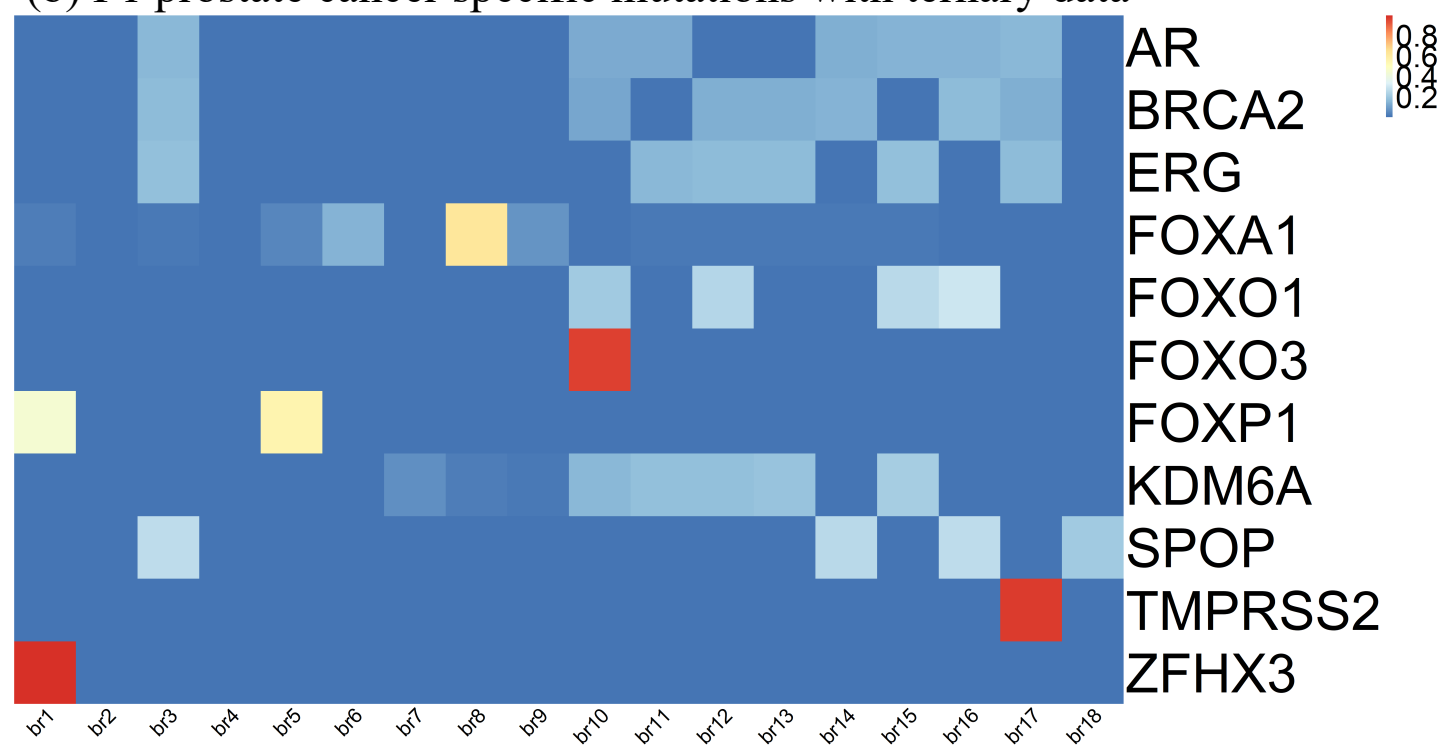

Fig. 12: Heatmap of posterior probabilities of mutation branch for P1 using (a) binary or (b) ternary data. This heatmap is for the prostate cancer-specific genes. Color indicates the magnitude of the probability, with red indicating probability close to 1 and blue indicating probability close to 0 . For P1, the prior for $\alpha$ is set to $\alpha \mid \mathbf{S}_{i} \sim \operatorname{Beta}(0 \cdot 29,0 \cdot 71)$ (larger variance). The prior for $\beta$ is set to $\beta \mid \mathbf{S}_{i} \sim \operatorname{Beta}(0 \cdot 02,0 \cdot 98)$ (larger variance). The distribution of transition rate $\lambda_{1}$ is set to $\lambda_{1} \mid \mathbf{S}_{i} \sim \operatorname{Gamma}\left(2,5 \cdot 0 \times 10^{-8}\right)$ (larger variance). The distribution of transition rate $\lambda_{2}$ is set to $\lambda_{2} \mid \mathbf{S}_{i} \sim \operatorname{Gamma}\left(2,5 \cdot 0 \times 10^{-3}\right)$ (larger variance) (cont.). 
(c) P2 prostate cancer specific mutations with binary data

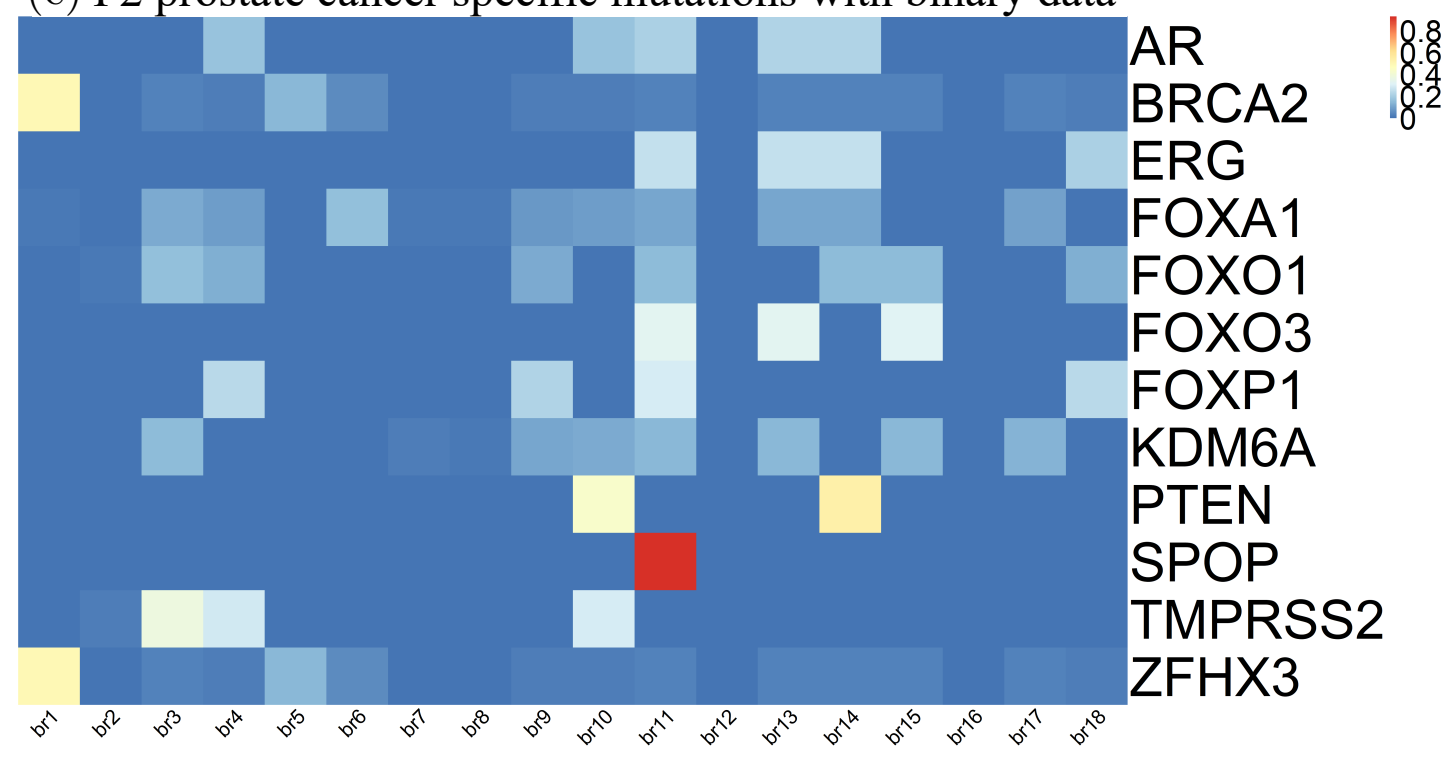

(d) P2 prostate cancer specific mutations with ternary data

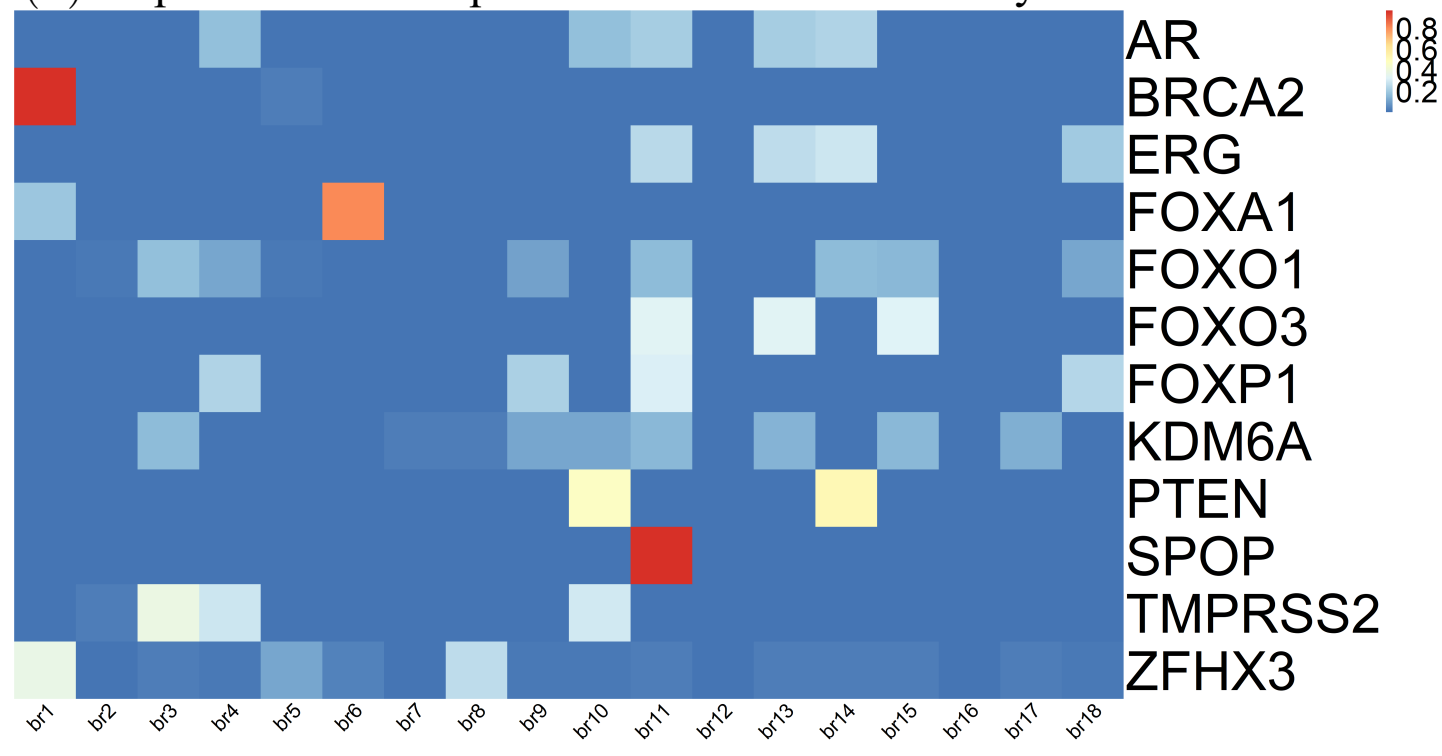

Fig. 12: Heatmap of posterior probabilities of mutation branch for P2 using (c) binary or (d) ternary data. This heatmap is for the prostate cancer-specific genes. Color indicates the magnitude of the probability, with red indicating probability close to 1 and blue indicating probability close to 0 . For P2, the prior distribution for $\alpha$ is set to $\alpha \mid \mathbf{S}_{i} \sim \operatorname{Beta}(0 \cdot 31,0.69)$ (larger variance). The prior distribution for $\beta$ is set to $\beta \mid \mathbf{S}_{i} \sim$ $\operatorname{Beta}(0 \cdot 02,0.98)$ (larger variance). The distribution of transition rate $\lambda_{1}$ is set to $\lambda_{1} \mid \mathbf{S}_{i} \sim \operatorname{Gamma}(2,5 \cdot 0 \times$ $10^{-8}$ ) (larger variance). The distribution of transition rate $\lambda_{2}$ is set to $\lambda_{2} \mid \mathbf{S}_{i} \sim \operatorname{Gamma}\left(2,5 \cdot 0 \times 10^{-3}\right)$ (larger variance). 